\title{
Hydrodynamical and radio evolution of young supernova remnant G1.9+0.3 based on the model of diffusive shock acceleration
}

\author{
M. Z. Pavlović ${ }^{1 \star}$ \\ 1 Department of Astronomy, Faculty of Mathematics, University of Belgrade, Studentski trg 16, 11000 Belgrade, Serbia
}

Accepted 2017 February 23. Received 2017 February 9; in original form 2016 December 28

\begin{abstract}
The radio evolution of, so far the youngest known, Galactic supernova remnant (SNR) G1.9+0.3 is investigated by using three-dimensional (3D) hydrodynamic modeling and non-linear kinetic theory of cosmic ray (CR) acceleration in SNRs. We include consistent numerical treatment of magnetic field amplification (MFA) due to resonant streaming instability. Under the assumption that SNR G1.9+0.3 is the result of a type Ia supernova explosion located near the Galactic centre, using widely accepted values for explosion energy $10^{51} \mathrm{erg}$ and ejecta mass $1.4 M_{\odot}$, the non-thermal continuum radio emission is calculated. The main purpose of the paper is to explain radio flux brightening measured over recent decades and also predict its future temporal evolution. We estimate that the SNR is now $\sim 120$ years old, expanding in ambient density of $0.02 \mathrm{~cm}^{-3}$ and explain its steep radio spectral index only by means of efficient non-linear diffusive shock acceleration (NLDSA). We also make comparison between simulations and observations of this young SNR, in order to test the models and assumptions suggested. Our model prediction of a radio flux density increase of $\sim 1.8$ per cent $\mathrm{yr}^{-1}$ during last two decades agrees well with measured values. We synthesize synchrotron spectrum from radio to X-ray and it fits well VLA, MOST, Chandra and NuSTAR measurements. We also propose simplified evolutionary model of the SNR in gamma-rays and suggest it may be a promising target for gammaray observations at $\mathrm{TeV}$ energies with the future generation of instruments like CTA. SNR G1.9+0.3 is the only known Galactic SNR with the increasing flux density and we present here the prediction that flux density will start to decrease approximately 500 years from now. We conclude this is a general property of SNRs in free expansion phase.
\end{abstract}

Key words: acceleration of particles - (ISM:) cosmic rays - hydrodynamics - ISM: individual objects: G1.9+0.3 - (ISM:) supernova remnants - radiation mechanisms: non-thermal

\section{INTRODUCTION}

A potentially young shell type Galactic supernova remnant (SNR) G1.9+0.3 was identified for the first time by Green \& Gull (1984), from Very Large Array (VLA) observations of a sample of small-diameter Galactic radio sources. The interest in this SNR has increased after the work of Reynolds et al. (2008) and Green et al. (2008) who deduced that G1.9+0.3 is of order 100 years old and therefore, the youngest SNR in the Galaxy. Based on extremely high absorption, they placed G1.9+0.3 near the Galactic centre

* Contact e-mail: marko@math.rs
(GC), at a distance of about $8.5 \mathrm{kpc}$, where the mean diameter would be about $4 \mathrm{pc}$, and the required expansion speed about $14,000 \mathrm{~km} / \mathrm{s}^{1}$. Roy \& Pal (2014) propose a lower limit on its distance from Sun as $10 \mathrm{kpc}$, based on GMRT measurements of absorption by known anomalous velocity features near the GC.

According to Reynolds et al. (2008), the synchrotrondominated X-ray spectrum clearly indicates that effective particle acceleration takes place, at least for electrons, given the very high shock velocities and low ambient densities.

1 Derived from both expansion proper motions and Doppler shifts of lines from isolated regions of thermal emission 
Also, the implied characteristic roll-off electron energy of about $100 \mathrm{TeV}$ is the highest ever reported for a shell SNR.

Borkowski et al. (2013) reported spatially resolved spectroscopy of SN ejecta, and interpreted their results in the framework of an energetic and asymmetric Type Ia explosion. They also concluded that outermost ejecta layers in free-expansion have velocities in excess of 18,000 km/s. Several arguments suggest a Type Ia origin of G1.9+0.3 (see also Reynolds 2008): the high velocities more than $100 \mathrm{yr}$ after the explosion; the absence of central pulsar-wind nebula; the bi-symmetric morphology in X-ray and substantial thermal emission from Fe. A usual core-collapse event could not reproduce the observations, while a SN Ia model can easily reach the observed size and velocity for a mean ambient density of about $0.02 \mathrm{~cm}^{-3}$ (Carlton et al. 2011).

Green et al. (2008) compared their VLA radio observations of the SNR G1.9+0.3 at $4.86 \mathrm{GHz}$ and $1.43 \mathrm{GHz}$ with earlier observations at $1.49 \mathrm{GHz}$ which have a comparable resolution. They found evidence that this SNR has been brightening over the last few decades in the radio emission at a rate of $\approx 2$ per cent $\mathrm{yr}^{-1}$ for the available flux densities and proposed explanation that the efficiency of particle acceleration and/or magnetic field amplification has been increasing. De Horta et al. (2014) analyzed all available radio-continuum observations of SNR $1.9+0.3$ at $6-\mathrm{cm}$ from the VLA and also Australia Telescope Compact Array (ATCA) (see Figure 1), obtaining results which are in broad agreement with the estimates of expansion made by Reynolds et al. (2008) and Green et al. (2008).

By using the time-dependent non-linear kinetic theory for cosmic ray (CR) acceleration in SNRs, coupled with 1D spherically symmetric gas dynamics, Berezhko \& Völk (2004) predicted that radio luminosity should increase during the free expansion phase, in general case. According to these authors, this is mainly due to the growing number of accelerated CRs. Ksenofontov et al. (2010) also applied similar model of non-linear CR acceleration to study the nonthermal properties of SNR G1.9+0.3. They obtained spatially integrated radio synchrotron flux slowly increases with time, explaining it as a consequence of the rapidly increasing total number of accelerated electrons in the increasing SNR volume $\propto R_{\mathrm{S}}^{3}$, where $R_{\mathrm{S}}$ represents current shock radius.

Recently, Chakraborti et al. (2016) used their analytical model to demonstrate that a double degenerate (DD) progenitor can explain the decades-long flux rise and size increase of the SNR G1.9+0.3 and disfavor a single degenerate (SD) scenario for this SNR. Nevertheless, for pre-explosion circumstellar density $\rho_{\mathrm{cs}} \propto r^{-s}$ they assume index $s=2$ for SD and $s=0$ for DD case, which may be questionable.

It should be examined whether the increasing radio brightness of G1.9+0.3 is a unique property among the SNRs in our Galaxy and does it require some special conditions. One of the aims will also be to go beyond the specific analysis of G1.9+0.3 and, in some future papers, expand proposed analysis to any young SNR or further to a global sample of SNRs with different ages.

Why we think the radio evolution modeling is important? Electrons emitting at a radio frequency have the acceleration time-scales of the order of a week, when the Bohm diffusion is assumed (Petruk \& Kopytko 2016). This is much less than the acceleration time of the highest-energy particles, for which it is of the order of an SNR age. Due

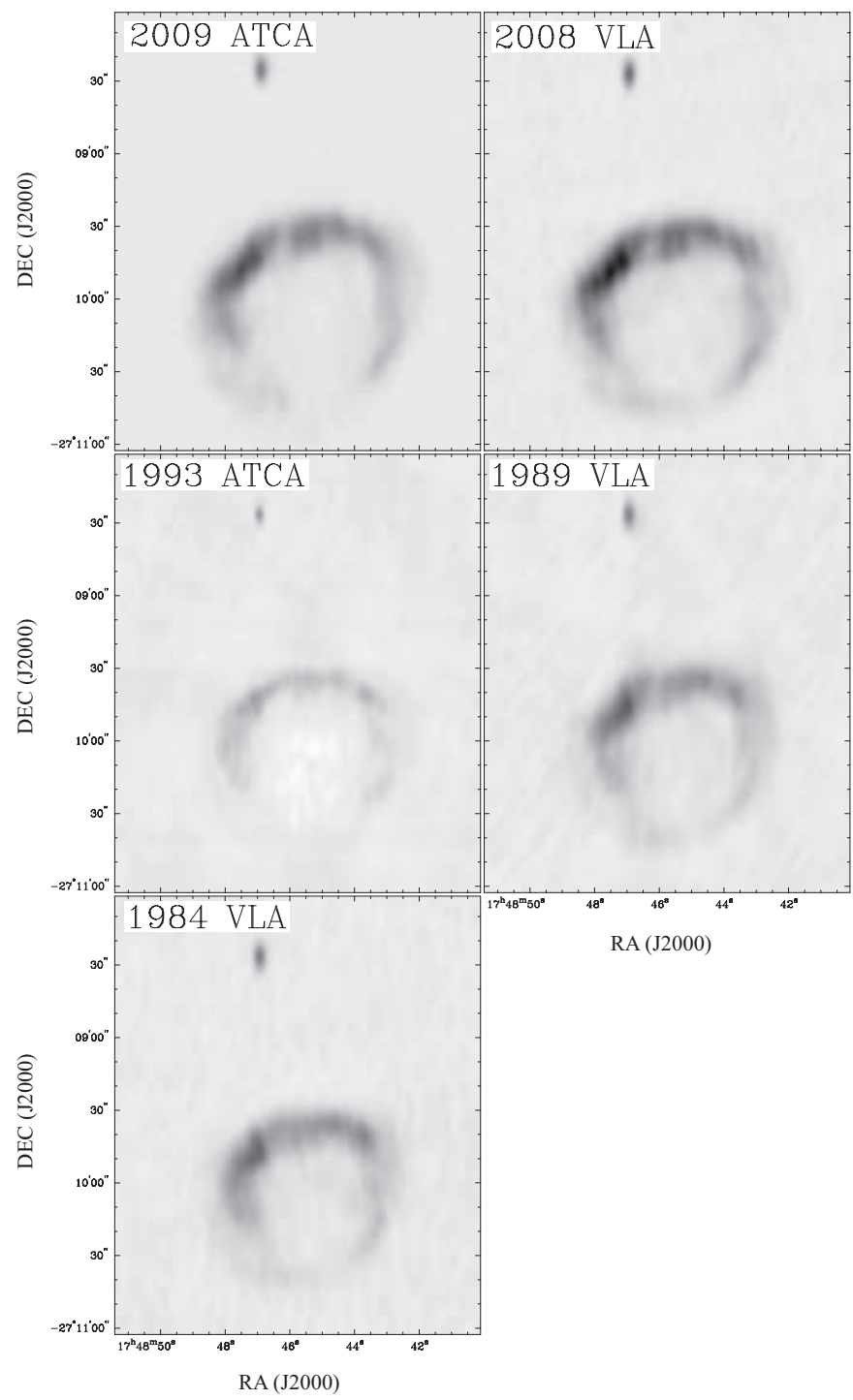

Figure 1. Matched resolution 6-cm images of Galactic SNR (centred at $\left.\mathrm{RA}(\mathrm{J} 2000)=17^{\mathrm{h}} 48^{\mathrm{m}} 45.4^{\mathrm{s}}, \operatorname{Dec}(\mathrm{J} 2000)=-27^{\circ} 10^{\prime} 06^{\prime \prime}\right)$ at multiple epochs. Left to right, top to bottom, 2009 ATCA, 2008 VLA, 1993 ATCA, 1989 VLA \& 1984 VLA. (De Horta et al. 2014)

to this, modeling of radio evolution and connecting it to the radio observations may reveal the present-time behavior of the injection efficiency and also its time dependence (Petruk \& Kopytko 2016).

Also, the radio surface-brightness-to-diameter $(\Sigma-D)$ relation for SNRs, being an useful distance determination tool, can be significantly improved if the radio evolution is better understood. This relation is known to be depend on properties of the SN explosion such as the explosion energy, mass of the ejected matter and also on the properties of ISM such as density, magnetic field strength, etc. One of the main shortcomings of this relation is the severe data scatter which is mainly due to the spread in mentioned parameters, in addition to measurement errors and selection effects (see for example Arbutina \& Urošević 2005; Urošević et al. 2010; Pavlovic et al. 2014, etc.). Theoretical considerations contain many limitations, because they often rely on simpli- 
fied assumptions about the evolutionary stage of SNRs, particle spectra and its evolution, magnetic field evolution etc. Numerical simulations should provide better understanding of underlying physics and explanation of the observed statistical properties.

Magnetic field is one of the main ingredients in particle acceleration and non-thermal emission. Consistent, timedependent calculation of magnetic field amplification (MFA) is one of the main advantages in approach based on numerical simulations.

\section{MODEL}

The dynamical evolution of an SNR was modeled by numerically solving the time-dependent hydrodynamical (HD) equations of mass, momentum and energy conservation, including a semi-analytical model of acceleration and back reaction of particles on shock dynamics. We threat the backreaction of the energetic particles on the shock in sense of the pressure of particles which affect shock dynamics (Blasi 2004) and cosmic ray (CR) current which amplifies the magnetic field (Bell 2004; Caprioli et al. 2009). Both effects happen upstream of the shock wave, in the so-called precursor. Accent of this paper is given to the spatially integrated radio emission of a single SNR and its temporal evolution, but also to the development of a consistent model which can be applied to any type Ia SNRs.

Through our paper, we will use "classical" non-linear diffusive shock acceleration (NLDSA), which naturally predicts spectrum steepens at low energies and flattens at higher energies, as the compression ratio felt by a diffusing particle depends on particle's energy. Recent gamma-ray observations of Galactic SNRs seriously challenge this understanding of $\mathrm{CR}$ acceleration at fast shocks. There are evidences of high-energy part of CR spectra steeper than $E^{-2}$ (significantly steeper than what is expected in NLDSA which is implemented in our model) coming mainly from gammaray observations of SNRs (Caprioli 2012). He developed a self-consistent scenario in which magnetic field amplification (MFA) induces the conditions for reversing previously mentioned trend and can lead to a steepening in the high energy part of spectrum of CRs. The crucial point in Caprioli (2012) is that he takes the Alfvén speed in the amplified magnetic field (as being $\delta B \gg B_{0}$ ) instead of calculation in the ambient field $B_{0}$. This is not crucial for the present study and not modeled here because the part of the electron spectrum that matters for radio emission is at much lower energies. As for higher energies, it should be included in our future modeling, especially if we are interested in gamma-ray emission produced in hadronic scenario, with spectrum and emissivity directly related to the spectrum of highest energy CRs. Simplified model of gamma-ray evolution, used in our paper, is not strongly influenced by the shape of the spectrum as it approximates it with $E^{-2}$.

\subsection{Hydrodynamic modeling}

The dynamical evolution of an SNR was modeled by numerically solving the time-dependent HD equations of mass, momentum and energy conservation:

$\frac{\partial}{\partial t}\left(\begin{array}{c}\rho \\ \rho \mathbf{v} \\ E\end{array}\right)+\nabla \cdot\left(\begin{array}{c}\rho \mathbf{v} \\ \rho \mathbf{v v}+\mathbf{I} P \\ (E+P) \mathbf{v}\end{array}\right)^{\mathrm{T}}=\left(\begin{array}{l}0 \\ 0 \\ 0\end{array}\right)$

where $\rho$ is the mass density, $\mathbf{v}$ the flow velocity, $P$ the thermal pressure, $\mathbf{I}$ the unit vector, $E$ the total energy density and $\gamma$ is the adiabatic index. The total energy density is a sum of the thermal and kinetic components:

$E=\frac{P}{\gamma-1}+\frac{1}{2} \rho v^{2}$

We adopt the PLUTO code (Version 4.2; Mignone et al. 2007, 2012) to solve the system of HD conservation laws by using a cell-centred finite-volume approach based on Godunov-type schemes. The code design enables efficient usage of massively parallel computers through the message passing interface standard (MPI) for interprocessor communications.

We do not include radiative cooling and thermal conduction in our HD equations, describing only the free and Sedov expansion phases of the SNR evolution in a tenuous, collisionless medium. The transition time from Sedov to radiative phase for an SNR is described with the approximation (e.g., Blondin et al. 1998; Petruk 2005; Orlando et al. 2011)

$t_{\text {tr }}=2.84 \times 10^{4} E_{51}^{4 / 17} n_{\mathrm{H}}^{-9 / 17} \mathrm{yr}$,

where $E_{51}=E_{0} /\left(10^{51} \mathrm{erg}\right)$ and $E_{0}$ initial total explosion energy, contained mostly in form of kinetic energy. In our set of simulations, $t_{\mathrm{tr}}>0.5 \mathrm{Myr}$ and therefore our modeled SNRs never reach the radiative phase. We will compute the radio emission from the SNR but with assumption that that radiation has no impact yet on its dynamical evolution.

Throughout our modeling, we do not activate the MHD solver of PLUTO because it is in any case powerless in describing the generation of magnetic turbulence by cosmic rays upstream of the shock and corresponding MFA. Such an amplified magnetic field is dominant compared to the field compressed only due to fluid compression, especially for young SNRs where non-linearity is very pronounced. NLDSA module, which runs parallel with PLUTO HD code, simultaneously performs calculations of MFA, synthesize the global radio emission in this amplified field and also accounts for its impact on hydrodynamics.

We used the following set of PLUTO algorithms in our simulations: linear interpolation with default limiter, HLLC Riemann solver and RK2 algorithm for the time evolution. Additionally, we used MULTID shock flattening algorithm for the numerical dissipation near the strong shocks. Our three-dimensional (3D) computations were carried out in spherical coordinates $(r, \theta, \phi)$, by using a static logarithmic grid, with mesh size increasing with the SNR radius.

Detection and tracking of the SNR shock waves in the fluid, traveling in some direction $x$, is based on two standard 
numerical conditions, namely $\nabla \mathbf{v}<0$ and $\Delta x \frac{\nabla P}{P}>\varepsilon_{\mathrm{p}}$, where $\varepsilon_{\mathrm{p}}$ represents parameter, setting the shock strength.

We modified PLUTO modules ${ }^{2}$ in order to couple the hydrodynamical evolution of the remnant with particle acceleration. Instead of a constant adiabatic gas index $\gamma$ (ratio of specific heats), obeying the ideal gas low $P=(\gamma-1) \boldsymbol{\epsilon}$, where $\epsilon$ represents thermal energy density, we adopted hydrodynamic equations to use the space and time-dependent adiabatic index $\gamma_{\text {eff }}=\gamma_{\text {eff }}(x, y, z, t)$ i. e. $P=\left(\gamma_{\text {eff }}-1\right) \epsilon$. The effective adiabatic index $\gamma_{\mathrm{eff}}$, defined so it produces the same total compression $R_{\text {tot }}$ as obtained from non-linear model (described later in section 2.2), is calculated at the shock front and then advected within the remnant, remaining constant in each fluid element as in Ellison et al. (2004) (see also Ferrand et al. 2010; Orlando et al. 2012). As pointed out by Ferrand et al. (2010, 2012), each fluid element should remember the effect of shock modification induced by accelerated CRs at the time when shock wave passes through the fluid element. To fulfill this requirement, we threat gas adiabatic index as PLUTO built-in code feature called "passive scalar" or "colour" (denoted by $Q_{k}$ ) obeying simple advection equation of the form

$$
\frac{\mathrm{D} Q_{k}}{\mathrm{D} t}=0,
$$

which is added to the standard set of hydrodynamic equations (Equation 1), where $\frac{\mathrm{D}}{\mathrm{Dt}}=\frac{\partial}{\partial t}+\mathbf{v} \cdot \nabla$ denotes Lagrangian time-derivative.

The shock precursor is not explicitly modeled in the hydrodynamic part of our simulations. Precursor properties are handled in a separate modules containing a non-linear acceleration calculation. Non-linear effects on SNR hydrodynamics are visible only through the effective adiabatic index, explained in the next section.

\subsection{Diffusive shock acceleration}

We model the evolution of an SNR, including the effect of the high-energy CR particles accelerated by the shock wave. It is widely accepted that the most efficient process of particle acceleration in SNRs is the diffuse shock acceleration (DSA), proposed by Bell (1978a,b) and Blandford \& Ostriker (1978). Also known as first-order Fermi mechanism ${ }^{3}$, it provides the energy gain due collisions with irregularities of the magnetic field of $\Delta E / E \propto u / v$, that is, first order in $u / v$, where $u$ is the velocity of magnetic perturbation and $v$ is the velocity of high-energy particle. For additional reviews of particle acceleration theories, see for example Reynolds (2008, 2011) and Urošević (2014).

In order to take into account the non-linear back reaction of accelerated particles on the fluid structure, we use the semi-analytical model of Blasi $(2002,2004)$ and Blasi et al. (2005). This model iteratively solves the particle distribution

\footnotetext{
2 By default, algorithms in the PLUTO code are explicitly based on the assumption of a constant gamma law.

3 Enrico Fermi's idea that particles gain energy in collisions with the moving irregularities of the magnetic field (Fermi 1949), provides the basis of modern acceleration theories, including DSA.
}

function $f(p)^{4}$ and the dimensionless fluid velocity $U(p)$, both as functions of particle momentum $p$. The boundary condition $U\left(p_{\max }\right)=1$ has to be fulfilled since at $p>p_{\max }$ there are no CRs to contribute any pressure. Function $U(p)$ represents quantity defined as $U(p)=u_{p} / u_{0}$, where $u_{p}$ represents the average fluid velocity experienced by particles with momentum $p$ while diffusing upstream away from the shock surface and $u_{0}$ is the fluid velocity far upstream (shock wave velocity).

In the following, we will use standard indexing of quantities around shock wave, namely subscript 1 (2) for parameters immediately upstream (downstream), while subscript 0 denotes undisturbed, far upstream quantities. We introduce three quantities $R_{\text {sub }}=u_{1} / u_{2}, R_{\text {tot }}=u_{0} / u_{2}$ and $R_{\text {pre }}=u_{0} / u_{1}$ which are respectively the compression factor at the gas subshock, the total compression factor and compression in the shock precursor. The compression ratio $R_{\text {sub }}$ $\left(R_{\mathrm{tot}}\right)$ is expected to be lower (higher) than for the standard test-particle (TP) case where $R=4$. For a strongly modified shock, $R_{\text {tot }}$ can attain values much larger than $R_{\text {sub }}$.

The model iteratively solves acceleration, by numerical integration of integro-differential equations, providing the values for shock compressions $R_{\text {sub }}, R_{\text {pre }}$ and $R_{\text {tot }}$ which give $U(p)$ solution, satisfying $U\left(p_{\max }\right)=1$. This model only finds quasi-stationary solutions, so that we have to rerun it after each hydro time step (see recent paper Petruk \& Kopytko (2016) which describes the time-dependent DSA at the nonrelativistic shocks). Having the overall compression ratio, $R_{\text {tot }}$, the effective ratio of specific heats is calculated as (Ellison et al. 2004)

$\gamma_{\mathrm{eff}}=\frac{M_{S, 0}^{2}\left(R_{\mathrm{tot}}+1\right)-2 R_{\mathrm{tot}}}{M_{S, 0}^{2}\left(R_{\mathrm{tot}}-1\right)}$,

where $M_{S, 0}$ represents sonic Mach number far upstream.

Originally proposed non-linear acceleration model of Blasi usually gives high levels of shock modification, as total shock compression factors may exceed $\sim 50-100$ (Amato \& Blasi 2005) and thus do not compare well with some observations, suggesting $R_{\text {tot }} \sim 7-10$ or slightly higher (see e.g., Völk et al. 2005). We assume that part of the energy in the form of turbulent Alfén waves, excited by energetic particles and responsible for the scattering of charged particles, is damped on the thermal gas and heats the gas in the upstream region. Our model uses non-adiabatic compression in the precursor proposed by Berezhko \& Ellison (1999)

$\frac{P_{p}}{P_{0}}=U_{p}^{-\gamma}\left(1+\zeta(\gamma-1) \frac{M_{S, 0}^{2}}{M_{A, 0}}\left(1-U_{p}^{\gamma}\right)\right)$,

which is caused by the Alvfén heating and significantly reduces the shock modification. Here, $P_{0}$ represents far upstream fluid pressure, $P_{p}$ and $U_{p}$ are respectively the fluid pressure and dimensionless fluid velocity at any point $x_{p}$ (for a given diffusion law $D(p)$, particles of momentum $p$

${ }^{4}$ By definition, $f(p)$ satisfies $d N=4 \pi p^{2} f(p) d p$, where $N$ represents number of particles per unit volume. The energy distribution $f(E)$ can be calculated as $f(E)=4 \pi p^{2} f(p) \frac{d p}{d E}$, giving $f(E) \propto p^{2} f(p)$ in relativistic regime where $E \propto p$. 
diffuse up to a distance $x_{p} \sim D(p) / u_{p}$ where the fluid velocity is $u_{p}$ ) and $\zeta \in[0,1]$ is a free parameter introduced by Caprioli et al. (2009). This parameter accounts fraction $\zeta$ of the energy transferred from CR streaming to MHD waves is dissipated as heat in the plasma by non-linear damping processes. The damping of the waves is mitigated for $\zeta<1$ and therefore allows magnetic field amplification (MFA) ${ }^{5}$.

We use a recipe for the injection of particles from the thermal pool, proposed by Blasi et al. (2005), and set the following fraction $\eta$ of particles entering the acceleration process from Maxwellian downstream thermal pool

$\eta=\frac{4}{3 \sqrt{\pi}}\left(R_{\mathrm{sub}}-1\right) \xi^{3} e^{-\xi^{2}}$,

which assumes that only particles with momentum $p_{\text {inj }} \geq$ $\left(\xi-u_{2} / c\right) p_{\text {th,2 }}$ can be involved in acceleration process, where $p_{\mathrm{th}, 2}=\sqrt{2 m_{\mathrm{p}} k T_{2}}$ represents mean downstream thermal momentum, $m_{\mathrm{p}}$ proton mass and $T_{2}$ is downstream temperature. The shift $u_{2} / c$ is due to the assumption that thermal particles in downstream have a Maxwellian spectrum in the fluid reference frame, while $p_{\text {inj }}$ is taken in the shock frame. The injection parameter $\xi$ strongly affects acceleration fraction $\eta$. Caprioli \& Spitkovsky (2014a) conclude from their kinetic simulations that for parallel non-relativistic shocks a fraction of about $10^{-3}-10^{-4}$ of the particles crossing the shock is injected into the DSA process and that the injection parameter is $\xi \simeq 3-4$.

While the thermal leakage may represent a viable way of parameterizing injection, it doesn't account for the dependence of ion injection on shock inclination, elaborated by Bell et al. (2011). Recent PIC simulations bring back again the dependence of ion injection on shock inclination (Caprioli et al. 2015). They show that ions are injected not by being heated and then leaking, but instead by specular reflection. A different dependence of $\eta$ on subshock compression is then hard to encompass in a formula similar to Equation 7. This refinement is well beyond the goal of this paper, but should be somehow encompassed in future modeling.

It is worth stressing that using Equation 7 introduces some kind of time dependence of the acceleration efficiency, as compression at the subshock is not constant during SNR evolution. This may be seen as an improvement in comparison with previous models setting $\eta=$ const, keeping in mind that it may be still artificial and a questionable simplification. Recently proposed, new theoretical model of time-dependent shock acceleration of particles (Petruk \& Kopytko 2016) shows that variable injection could be a crucial element in explaining the X-ray and gamma-ray spectra of young SNRs, but not so important for radio. Nevertheless, the time dependence in injection efficiency is hard to model, and even more how proton-toelectron ration (introduced latter in Section 2.4) depends on time. Kinetic simulations, providing us first-principles calculations, seem to show that ion injection does not vary as long as the shock is strong, while the electron injection efficiency in the regimes considered here is still questionable.

5 Otherwise, if we have $\zeta \simeq 1$, the rate of damping of the waves is close to that of wave-growth and the MFA is heavily suppressed.
We assume that the CR distribution vanishes at a distance $\sim \chi_{\mathrm{esc}} R_{\mathrm{S}}$ upstream of the shock wave, where parameter $\chi_{\text {esc }}<1$ is the fraction of the shell radius $R_{\mathrm{S}}$, to account for the presence of a free-escape boundary beyond which highest energy CRs, mainly consisting of protons, cannot diffuse back at the shock and escape into the interstellar medium (ISM) (Caprioli et al. 2010; Morlino \& Caprioli 2012). This approximation allows us to determine the maximum momentum $p_{\mathrm{p}, \max }$ of accelerated protons by assuming

$\frac{D\left(p_{\mathrm{p}, \max }\right)}{u_{0}}=\chi_{\mathrm{esc}} R_{\mathrm{s}}$,

where $D(p)$ is Bohm-like diffusion constant i.e. $D(p)=$ $\frac{1}{3} v(p) r_{\mathrm{L}}(p)$, with $v(p)$ and $r_{\mathrm{L}}(p)$ are respectively the particle velocity and the Larmor radius, in agreement with the approach of Bell et al. (2013) and references therein. We use $\chi_{\text {esc }}=0.1$ in our model as suggested by Morlino \& Caprioli (2012), which satisfies condition that the acceleration time up to $p_{\mathrm{p}, \max }$ is less than the age of the system (Blasi et al. 2007).

\subsection{Magnetic field amplification}

Galactic cosmic-ray (CR) acceleration to the knee in the spectrum at a few $\mathrm{PeV}$ is only possible if the magnetic field ahead of an SNR shock is strongly amplified by CRs escaping the SNR (Bell et al. 2013). Consistent calculation of the magnetic field strength, although not yet fully understood, is of the utmost importance for the radio emission of energetic electrons. Due to this, we include MFA in our model.

The MFA is driven by streaming instabilities, induced by CRs in the vicinity of SNR shocks. Instabilities can be resonant (Bell 1978a), assuming that Alfvén waves, generated by particles streaming faster than Alfvén speed, have a wavelength in resonance with the $\mathrm{CR}$ Larmor radius, and strongly driven, nearly purely growing, non-resonant (Bell 2004). Non-resonant instabilities are not accurately described as Alfvén waves and they grow preferentially at wavelengths that are not resonant with the CR Larmor radius. Amato \& Blasi (2009) showed that the non-resonant modes are bound to be relevant mostly in the earlier stages of the SNR evolution, namely free expansion and early SedovTaylor, while streaming instability should be dominated by resonant waves for the most of the history of the SNR. However, according to Caprioli \& Spitkovsky (2014b), their equation obtained for resonant instability fits well up to simulations with $M_{A}=100$ and can also be extrapolated to higher Mach numbers, inferred at the blast waves of young SNRs, in case of efficient CR acceleration, which is certainly the case for G1.9+0.3. For such high- $M_{\mathrm{A}}$ shocks, according to Caprioli \& Spitkovsky (2014b), we can distinguish two regions: the far upstream region dominated by nonresonant instability, and the precursor, where resonant and non-resonant instabilities grow at a comparable rate. Closer to the SNR shock, resonant instabilities seems to take over but ambient magnetic field is already considerably modified in precursor. It is however, hard to simulate what happens for $M_{A} \gg 100$ in global shock simulations mentioned, as simulations become quite expensive, and lot of questions remain open here. Due to this, whichever model we choose to implement, it will contain a lot of uncertainties until the future 
PIC simulations and better theory give us improved understanding.

We choose to model MFA due to resonant streaming instability, being already compatible with Blasi's formalism and easy to implement in our code. With assumption that all the turbulence is generated via streaming instability in precursor, it is described by Caprioli et al. (2009) in form

$\frac{P_{\mathrm{w}, p}}{\rho_{0} u_{0}^{2}}=\frac{1-\zeta}{4 M_{A, 0}} U_{p}^{-3 / 2}\left(1-U_{p}^{2}\right)$,

This model of MFA is also used by Lee et al. (2012) and Ferrand et al. (2014). Here, $P_{\mathrm{W}}=\frac{1}{8 \pi}\left(\sum_{\mu} \delta B_{\mu}\right)^{2}$ denotes precursor magnetic pressure of Alfvén waves (subscript $\mu$ indicates modes of the magnetic turbulence) and $M_{A, 0}=u_{0} / v_{\mathrm{A}}=$ $u_{0} \sqrt{4 \pi \rho_{0}} / B_{0}$ is Alfvénic Mach number far upstream. For simplicity, we do not include pre-existing magnetic turbulence in the interstellar medium (ISM) in our models. The factor $(1-\zeta)$ is introduced to balance the factor $\zeta$ in Equation 6 , and accounts for local wave dissipation and reduction of MFA. The total magnetic field at point $x_{p}$ is then calculated with

$B_{p}^{2}=B_{0}^{2}+8 \pi P_{\mathrm{w}, p}$,

with $B_{0}$ denoting the ordered component of the ambient magnetic field. However, have in mind that this is rough estimate as effective ambient $B_{0}$ field that we need in the precursor is always determined by the Bell's non-resonant instabilities far upstream and will be larger than the few $\mu \mathrm{G}$ of the Galactic field.

The magnetic pressure in the amplified fields becomes quite high, comparable with or even higher than the thermal one, making the dynamical role of amplified magnetic fields non-negligible (Caprioli et al. 2009). Due to this, magnetic pressure as well as the pressure of accelerated particles are accounted for in NLDSA part of the presented calculations and then affect fluid compressibility through effective adiabatic index. The global shock modification follows from the conservation of momentum between the far upstream medium and any precursor point $x_{p}$, which involves 4 terms: dynamical pressure $\rho u^{2}$, thermal pressure $P$, nonthermal pressure of CRs computed from their distribution $f(p)$ as $P_{\mathrm{cr}}=\frac{4 \pi}{3} \int p^{2} f(p) p v(p) d p$ and magnetic pressure $P_{\mathrm{w}}$ (Ferrand et al. 2014); described with equation

$\rho_{p} u_{p}^{2}+P_{\mathrm{th}, p}+P_{\mathrm{cr}, p}+P_{\mathrm{w}, p}=\rho_{0} u_{0}^{2}+P_{\mathrm{th}, 0}+P_{\mathrm{cr}, 0}+P_{\mathrm{w}, 0}$.

As we will see later, the factor $\zeta$ in our simulations is close to $1 / 2$ and therefore gives the amplified magnetic field important role in SNR dynamics.

Also note that magnetic field amplification leads to nonadiabatic heating of the background plasma (turbulent heating, mentioned in Section 2.2), in such a way that plasma and magnetic field pressure are almost in equipartition throughout the precursor (Caprioli \& Spitkovsky 2014a).

The maximum value of the amplified upstream magnetic field $B_{1}$ is reached immediately ahead of the shock wave and we calculate it by putting $U_{p}=u_{1} / u_{0}=1 / R_{\text {pre }}$ in Equation 9. We use a common assumption that the Bfield is totally random in orientation, as a consequence of the strong turbulence. We then assume that downstream magnetic field is compressed only due to fluid compression such that the components in a shock plane are compressed and the three components of the magnetic field are roughly equal. The downstream magnetic field is then given by $B_{2}=B_{1} \sqrt{1 / 3+2 / 3 R_{\text {sub }}^{2}}$.

We also account for damping of the amplified magnetic field in the downstream region. We use the following recipe for the downstream magnetic field (Morlino \& Caprioli 2012), based on the non-linear Landau damping mechanism

$B_{d}(r) \simeq B_{2} \exp \left(-\frac{R s-r}{\lambda_{\mathrm{nl}}}\right)$,

The typical length scale $\lambda_{\text {nl }}$ for the non-linear Landau damping given by

$\lambda_{\mathrm{nl}}=\frac{3 \chi_{\mathrm{esc}}}{0.05} \frac{u_{0}^{2}}{v_{A}\left(B_{2}\right) c} R_{s}$

where $v_{A}\left(B_{2}\right)$ is Alfvén velocity in downstream region.

Bell's model for the MFA due to the non-resonant streaming instability (Bell 2004) predicts the total saturated magnetic energy density

$\frac{B_{\mathrm{sat}}^{2}}{2 \mu_{0}} \sim \frac{1}{2} \frac{u_{0}}{c} \epsilon_{\mathrm{cr}}$

where $\boldsymbol{\epsilon}_{\mathrm{cr}}$ represent the CR energy density at the shock. In the active SNR phase, when $\epsilon_{\mathrm{cr}} \sim \rho_{0} u_{0}^{2}$, this results in an amplified magnetic field of $B \propto u_{0}^{3 / 2}$. Another point of view, namely equipartition between the total energy densities of cosmic rays and that of the magnetic field (Beck \& Krause 2005; Arbutina et al. 2012), results in the $B \propto u_{0}$. Both dependencies, mentioned above, were implemented in the numerical model of Ksenofontov et al. (2010), while Berezhko \& Völk (2004) used only the later one. Vink (2012) favors a dependency $B \propto u_{0}^{3 / 2}$, providing the observational evidence, but also notes that the dynamic range makes the dependency on shock velocity uncertain. On the other hand, $B \propto u_{0}^{3 / 2}$ implementation in Ksenofontov et al. (2010), results in a too slow increase in radio flux density of SNR G1.9+0.3 to provide a reasonable agreement with observations.

Equation 9, used as a receipt for MFA in our modeling of radio evolution, is a pretty much non-linear and therefore the magnetic filed dependence on shock velocity will be affected by other simulation parameters. If we deduce from Equation 9 that $P_{\mathrm{w}, p} \sim \frac{\rho_{0} u_{0}^{2}}{M_{A, 0}}$, we obtain $B \propto\left(u_{0} B_{0}\right)^{1 / 2}$ which should be taken with caution as the term $U_{p}^{-3 / 2}\left(1-U_{p}^{2}\right)$ probably brings additional dependence on shock velocity. We will see later in Section 3 the relation between amplified magnetic field and shock velocity, obtained from our simulations.

Previous paragraph demonstrates important difference between MFA driven by resonant and non-resonant streaming instabilities. Saturation of the resonant CR-driven instability explicitly depends on initial $B_{0}$, which is not the case for Bell's non-resonant instabilities (see, for example, equations 12 and 13 in Amato 2011).

Magnetic field modeling here do not consider its stretching and amplifying caused by the initial clumping of the 
ejecta and due to the development of Rayleigh-Taylor instabilities (see for example Orlando et al. 2012). It is thus important to simulate these effects by using PLUTO MHD in future, in order to follow morphological evolution of SNR along with the integrated radio emission which is modeled here.

\subsection{Radio emission}

Although the injection mechanisms for electrons are much less clear than for protons, we use assumption that electron injection is the same as that of protons and normalize their spectrum with respect to the protons' spectrum:

$f_{\mathrm{e}}(p)=K_{\mathrm{ep}} f_{\mathrm{p}}(p)$,

where parameter $K_{\text {ep }}$ represents the electron-to-proton ratio, very likely related to the different mechanisms responsible for lepton and hadron injection. We allow this parameter to vary from the value $K_{\mathrm{ep}}=10^{-2}$, observed near Earth in the diffuse spectrum of Galactic CRs around $\mathrm{GeV}$ energies. Since the energy losses of $\mathrm{GeV}$ electrons are not significant for propagation in the Galaxy, the electron to proton ration is also about $10^{-2}$ in the source. Nevertheless, this does not mean that this ratio must be the same for young SNRs (Zirakashvili 2008). Young SNRs, like G1.9+0.3, may be the main source of Galactic electrons with energies higher than $10 \mathrm{TeV}$, while $\mathrm{GeV}$ electrons may be produced in older SNRs and therefore, the value measured in CRs seems to be determined by later stages of the SNR evolution (e.g., Sarbadhicary et al. 2017). From recent PIC simulations of simultaneous acceleration of protons and electrons (Park et al. 2015), CR electron to proton ratio is inferred to be $K_{\text {ep }} \approx 10^{-3}-10^{-2}$, for shock velocity $u_{0} / c \approx 0.02-0.1$ and reduced electron to proton mass $m_{\mathrm{p}} / m_{\mathrm{e}}$ ranging from 100 to $400^{6}$. We assume the spectrum of accelerated electrons is parallel to protons' one, except for large momenta, since DSA mechanism should not be dependent on charge. We neglect the dynamical role of electrons in our model.

Assumption about parallel proton and electron spectra holds as long as synchrotron losses are neglected. However, at energies around $\mathrm{TeV}$, electrons suffer synchrotron losses which can be consistently taken into account by supplementing the ordinary diffusive transport equation by a corresponding loss term (as done for example in Berezhko et al. 2002; Berezhko \& Völk 2004). Strict numerical treatment of electron cooling is beyond the scope of this paper, as for radio emitting electrons it can be safely neglected. Nevertheless, we implement simple "toy" model, dealing with high energy part of electron spectrum, in order to obtain satisfying model of synchrotron spectra from radio to X-ray domain, which is later demonstrated in Section 3. We assume that electron spectra above a certain energy becomes steeper i.e. changes from $p^{-q}$ to $p^{-(q+\delta)}$ (Tanaka et al. 2008; Longair 2011) and allow $\delta$ to be different than 1. Following

6 Authors find marginal change of $K_{\mathrm{ep}}$ when increasing $m_{\mathrm{p}} / m_{\mathrm{e}}$ from 100 to 400
Tanaka et al. (2008), we calculate the position of the transition from an uncooled to a cooled regime as

$E_{b}=1.25\left(\frac{B_{2}}{100 \mu \mathrm{G}}\right)^{-2}\left(\frac{t_{0}}{10^{3} y r}\right)^{-1} \mathrm{TeV}$,

where $t_{0}$ represents current SNR age. Going from $E_{b}$ (corresponding momentum $p_{b}$ ) to higher energies, we steepen previously calculated electron spectra and apply the following form of cut-off $\exp \left[-\left(p / p_{\mathrm{e}, \max }\right)^{2}\right]$, as suggested by Zirakashvili \& Aharonian (2007) for the loss dominated case.

We calculate electron maximum momentum $p_{\mathrm{e}, \max }$, in the Bohm diffusion regime, by using the approximate implicit expression, determined by Morlino et al. (2009). This approach is based on equating the acceleration time with the minimum between the time for energy losses and the age of the SNR, when only synchrotron losses are important,

$p_{\mathrm{e}, \max }=\frac{3}{2} \sqrt{\frac{m_{e}^{3} c^{4}}{e B_{1} r_{0}}} \frac{u_{0}}{c} U_{p}\left(p_{\mathrm{e}, \max }\right) \sqrt{\frac{1-R_{\mathrm{tot}}^{-1} U_{p}^{-1}\left(p_{\mathrm{e}, \max }\right)}{1+R_{B} R_{\mathrm{tot}} U_{p}\left(p_{\mathrm{e}, \max }\right)}}$,

where $r_{0}$ is the classical electron radius, $R_{B}$ is the magnetic field compression factor at the sub-shock.

Spectrum for electrons at the shock is then

$f_{\mathrm{e}, 0}(p)=K_{\mathrm{ep}} f_{\mathrm{p}, 0}(p) p^{-\delta} e^{-p^{2} / p_{\mathrm{e}, \max }^{2}}$,

where $\delta=0$ for momenta $p<p_{b}$ and $\delta>0$ for $p \geq p_{b}$. We do not expect a sharp break in the energy spectra for the electrons, but rather some steepening (Blasi 2010). Due to this, for our "toy" model, $\delta$ has to be continuous function of momenta, which is later obtained as the best-fitting to the observed spectra. Standard assumption has been applied, that the spatial distribution of particles for a plane shock is constant downstream and drops exponentially in the upstreamReynolds (2008). Adiabatic losses are not taken into account as we assume that most of the radio emission comes from the relatively thin shell near FS.

The total volume emissivity (power per unit frequency interval per unit volume) of the relativistic electrons is defined as

$\varepsilon_{v}=\int_{E} P(v) N(E) d E$.

Here, $P(v)$ is the total emissivity of a single electron of energy $E$ which has a pitch angle $\vartheta$ with respect to the magnetic field given by (Wilson et al. 2013)

$P(v)=\frac{\sqrt{3} e^{3} B \sin \vartheta}{m_{e} c^{2}} F\left(\frac{v}{v_{c}}\right)$

where $v_{c}=\frac{3 e B_{\perp} p^{2}}{4 \pi\left(m_{e} c\right)^{3}}$ is the electron critical frequency and $F(x)$ is a synchrotron function defined as

$F(x)=x \int_{x}^{\infty} K_{5 / 3}\left(x^{\prime}\right) d x^{\prime}$,

$K_{5 / 3}(x)$ being the modified, non-integer order Bessel function (Abramowitz \& Stegun 1972). With an accuracy of less than $0.6 \%$, synchrotron function $F(x)$ can be approximated with 
a linear combination of its known approximations for $x \ll 1$ and $x \gg 1$, derived by Fouka \& Ouichaoui (2013).

Hence, by combining these relations, SNR total luminosity at frequency $v$ is calculated from the obtained electron spectrum $f_{\mathrm{e}, 0}(p)$ by using the following expression

$L_{v}=\frac{16 \pi^{2} \sqrt{3} e^{3}}{m_{e} c^{2}} \int_{R_{\mathrm{cd}}}^{R_{s}} B_{\perp} r^{2} d r \int_{p_{\text {inj }}}^{p_{\mathrm{e}, \max }} p^{2} f_{\mathrm{e}, 0}(p) F\left(\frac{v}{v_{c}}\right) d p$,

where $B_{\perp}$ is the magnetic field component perpendicular to the line of sight $(\mathrm{LoS})$ and we shall use $B_{\perp}(r)=0.5 B_{d}(r)$.

Our model uses a reasonable approximation that the radio emission from accelerated electrons comes only from the shocked ISM located between contact discontinuity (at radius $R_{\mathrm{cd}}$ ) and forward shock (FS). This assumption is based on the fact that, because of compression of magnetic field in downstream, the overall synchrotron radiation of the SNR is dominated by the emission originating from downstream region.

In order to obtain the radio flux density $S_{v}$ at given SNR distance $d$, we use the following relation:

$S_{v}=\frac{L_{v}}{4 \pi d^{2}}$

Many authors have implemented an idea that CR acceleration may also occur at the reverse shock (RS) of young SNRs (Ellison et al. 2005; Zirakashvili \& Aharonian 2010; Zirakashvili \& Ptuskin 2012, and others) although it is hard to expect a very large magnetic field in the ejecta into which RS propagates. The magnetic field frozen in the ejecta dilutes orders of magnitude below levels required to accelerate particles, during the early expansion phase of SNR. Gotthelf et al. (2001) have identified the FS and RS in Cassiopeia $\mathrm{A}$ and showed that radio (together with $\mathrm{Si}$ ) emissivity radial profile show a sharp rise at what they characterize as the RS. However, Morlino \& Caprioli (2012) concluded that there is no evidence of DSA at the RS, for the particular case of relatively young Tycho SNR, by investigating its radial profile of the radio emission. Similar to the later case, currently available VLA and ATCA radial profiles of G1.9+0.3 radio emission (Green et al. 2008; De Horta et al. 2014), taken between 1984 and 2009, do not show any emission which could be linked with the RS. Therefore, in this paper we only account for the radio emission from FS of SNR G1.9+0.3 and it seems enough to account for the observed radio flux.

\subsection{Simple estimates of the gamma-ray emission}

During the last decade, new generations of gamma-ray telescopes operating in $\mathrm{GeV}$ and $\mathrm{TeV}$ range, provided us new insights into SNR phenomenology and CR acceleration. It is generally accepted that two distinct physical mechanisms are responsible for gamma-emission. Electrons produce a gamma-radiation via inverse Compton (IC) scattering on different microwave, IR and optical photons, in the so-called leptonic scenario. The contribution of relativistic bremsstrahlung is also produced in leptonic scenario, but it is usually negligible in SNRs. In the second, so-called hadronic scenario, gamma-rays are produced by the decay of neutral pions $\left(\pi^{0}\right)$ produced in collisions between CRs and the background gas.
Despite relatively deep exposures, the High Energy Stereoscopic System (H.E.S.S.) data did not show any signs of significant $\mathrm{TeV}$ gamma-ray emission from SNR G1.9+0.3 (H.E.S.S. Collaboration et al. 2014).

As pointed out by Ksenofontov et al. (2010), the TeV gamma-ray flux is expected to increase with time as well as the radio flux, mainly due to the increase of overall number of CRs with energy above $10 \mathrm{TeV}$. It will be interesting to estimate the gamma-ray luminosity of the SNR, as a function of time and whether it could be visible in $\mathrm{TeV}$ gamma-rays by future instruments like the Cherenkov Telescope Array (CTA).

Consistent calculation of broadband gamma-emission of G1.9+0.3 is far from the main scope of this paper. Due to this, we will rather use simplified model of Zirakashvili (2008) which assumes $E^{-2}$ spectrum of highest energy protons at the shock front. This may not be so crude estimate, as Caprioli (2012) noted that slope predicted by the standard NLDSA is even farther from required $E^{-2.1}-E^{-2.2}$ to account for the observed gamma-ray phenomenology.

The differential gamma-ray flux from the pion decay at energies in the range $m_{p} c^{2}<E<0.1 E_{\mathrm{p}, \max }$ (where $E_{\mathrm{p} \text {,max }}$ represents maximum proton energy) may be estimated as proposed by Zirakashvili (2008)

$E^{2} F_{p p}(E)=\frac{R_{s}^{3} K_{\pi \pi} \sigma_{\mathrm{pp}} c n_{\mathrm{H}}^{2} \xi_{\mathrm{cr}} m_{p} u_{0}^{2}}{d^{2} \ln \left(p_{\mathrm{p}, \max } / m_{p} c\right)}\left(1+4 \frac{n_{\mathrm{He}}}{n_{\mathrm{H}}}\right)^{2}$.

Here $K_{\pi \pi}=0.17$ is the fraction of the proton energy transmitted to the parent neutral pions, $\sigma_{\mathrm{pp}}$ is the total inelastic $p-p$ cross-section, $\xi_{\mathrm{cr}}$ is the ratio of the cosmic ray pressure downstream of the shock $P_{\mathrm{cr}}$ to the dynamical pressure $\rho_{0} u_{0}^{2}$, while $n_{\mathrm{H}}$ and $n_{\mathrm{He}}$ represents hydrogen and helium number density in ISM respectively, assumed to be partitioned as $n_{\mathrm{H}}: n_{\mathrm{He}}=9: 1$. We use the value $\sigma_{\mathrm{pp}}=37.4 \mathrm{mb}$ for our estimate of $4 \mathrm{TeV}$ gamma-rays flux (Kelner et al. 2006). It was assumed that the accelerated protons fill the SNR uniformly.

The differential flux of gamma-rays from the IC scattering in the synchrotron losses dominated case may be estimated as proposed by Zirakashvili (2008)

$E^{2} F_{I C}(E)=\frac{3 \xi_{c r}}{8 \xi_{B}} \frac{K_{\mathrm{ep}} R_{s}^{2} U_{\mathrm{rad}} u_{0}}{d^{2} \ln \left(p_{\mathrm{p}, \max } / m_{p} c\right)}$.

Here $\xi_{B}$ is the ratio of the magnetic energy $B^{2} / 8 \pi$ to the dynamical pressure $\rho_{0} u_{0}^{2}$ and $U_{\text {rad }}$ is the energy density of the scattered photons, computed here only for cosmic microwave background $(\mathrm{CMB})$ radiation $U_{\mathrm{rad}}=(4 \sigma / c) T^{4} \approx$ $4.2 \times 10^{-13} \mathrm{erg} / \mathrm{cm}^{2} \mathrm{~s}$. Flux given in Equation 25 is valid for energies smaller than the cut-off energy given by

$E_{c} \approx 5 \mathrm{TeV}\left(\frac{\mathrm{u}_{0}}{3000 \mathrm{~km} / \mathrm{s}}\right)^{2}\left(\frac{\mathrm{B}_{2}}{100 \mu \mathrm{G}}\right)^{-1} \frac{\mathrm{T}}{2.7 \mathrm{~K}}$,

where $T$ represents the temperature of the scattered photons. We checked a posteriori that the gamma-ray energy around few $\mathrm{TeV}$ satisfies this requirement up to around 2500 years of simulated SNR evolution. 


\section{RESULTS AND DISCUSSION}

VLA observations from 1985 (Green 2004) show a strong asymmetry in the shell at $21 \mathrm{~cm}$, perhaps indicative of an external density gradient. The mean radius of the bright $\mathrm{X}$ ray ring is about $2 \mathrm{pc}$, but with the east and west "ears" at about 2.2 pc (Reynolds et al. 2008). We neglect any possible gradient of ambient density in surrounding ISM, which leads to a complicated morphology (see Orlando et al. 2007) and seek for a global qualitative description of integrated continuum radio emission.

Trying to model the observed radio morphology as a consequence of hypothetical global magnetic field gradient would be tricky, due to the existing disagreement between observations and theory. Young SNRs have a predominantly radial magnetic field structure, visible through polarization measurements (Reynolds et al. 2012; Helder et al. 2012). On the other side, modern theories and simulations of MFA predict a strong turbulence of amplified field (Bell 2004; Caprioli \& Spitkovsky 2014b), as a result of the interaction of CRs with the upstream plasma and ambient field.

We performed 3D HD simulations describing the expansion of the SNR G1.9+0.3 in a spherical coordinates with the PLUTO code, adopted according to the model described in previous section. As the magnetic field does not play a dynamical role in the evolution of the SNR, we do not use MHD modules existing in PLUTO. However, we calculate magnetic field strength and its amplification by using our separate NLDSA modules tied to PLUTO, as it is necessary for $\mathrm{CR}$ acceleration as well as for the radio emission.

Our initial conditions were chosen in order to reproduce G1.9+0.3 after around 100 yr of evolution ${ }^{7}$ in terms of shock radius, which is about 2 pc (near $100^{\prime \prime}$ in diameter) for an assumed location near the Galactic entre (Reynolds 2008), and shock velocity of $14,000 \mathrm{~km} \mathrm{~s}^{-1}$, deduced mainly from Fe emission with a width of about 28,000 $\mathrm{km} \mathrm{s}^{-1}$ (Borkowski et al. 2010). In all of our simulations, the ambient magnetic field strength is set to value $B_{0}=5 \mu \mathrm{G}$, representative of the average Galactic field. For the initial density structure of the ejecta, we used the exponential profile that has been shown to be the best approximate representation of explosion models for type Ia SNe (Dwarkadas \& Chevalier 1998), thought to represent thermonuclear disruption of a white dwarf. We add clumps in the initial ejecta as per-cell random density perturbations (Orlando et al. 2012) and they trigger Rayleigh-Taylor instability at the contact discontinuity. We assumed an initial spherical remnant with radius of $0.05 \mathrm{pc}$, (corresponding to an initial age of $\approx 2.5 \mathrm{yr}$ ), ejecta mass equal to the Chandrasekhar mass $M_{\mathrm{ej}}=1.4 M_{\odot}$ and a total explosion energy $E_{0}=10^{51} \mathrm{erg}$. SNR expands through a homogeneous isothermal plasma with temperature $T=10^{4} \mathrm{~K}$ (corresponding to an isothermal sound speed $c_{\mathrm{S}}=9.9$ and Alvén speed $v_{\mathrm{A}}=39 \mathrm{~km} / \mathrm{s}^{8}$ for ambient density $0.02 \mathrm{~cm}^{-3}$ ) and mass density $\rho_{0}=\mu m_{\mathrm{H}} n_{\mathrm{H}}$, characterized by the hydrogen number density $n_{\mathrm{H}}$, where $\mu=1.4$ is the mean atomic mass (assum-

7 De Horta et al. (2014) put the highest upper age limit of 180 years, assuming a constant expansion rate since the SN event.

8 Velocity $14000 \mathrm{~km} / \mathrm{s}$ therefore gives far upstream sonic Mach number $M_{\mathrm{S}} \approx 1410$ and Alfvénic number $M_{A} \approx 360$.

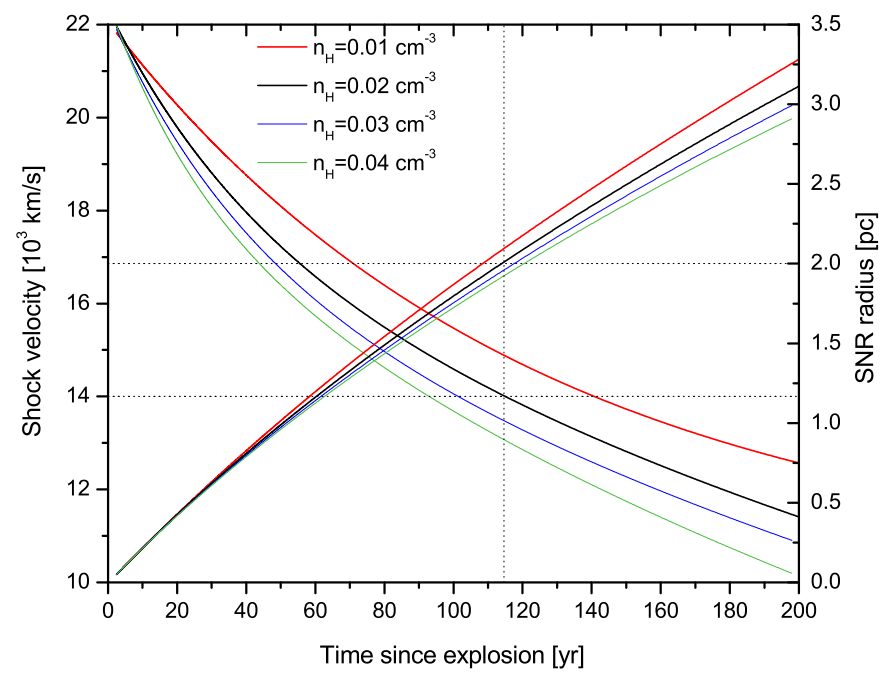

Figure 2. Evolution of an G1.9+0.3 shock velocity and radius, obtained from 3D HD simulations including efficient DSA. Left axis represents shock velocity in units of $10^{3} \mathrm{~km} / \mathrm{s}$, which is decreasing function of time. Right axis represents the radius of expanding SNR in parsecs. Radius and shock velocity evolution curves correspond to ISM hydrogen number densities of 0.01 (red thick line), 0.02 (black), 0.03 (blue) and 0.04 (green) $\mathrm{cm}^{-3}$. Horizontal dotted lines correspond to currently available measurements of mean radius and shock velocity from 2008. Vertical dotted line marks the epoch $t_{\mathrm{SNR}}=115 \mathrm{yr}$ when observed and simulated radius and shock velocity coincide i.e. represents our inferred age of the SNR. The starting time and radius for the simulation are respectively $t_{0} \approx 2.5$ yr and $R_{0} \approx 0.05 \mathrm{pc}$.

ing cosmic abundances) and $m_{\mathrm{H}}$ is the mass of the hydrogen atom.

In order to estimate ISM density and age of SNR, we performed a set of 3D HD simulations with different ambient hydrogen number densities ranging from $0.01 \mathrm{~cm}^{-3}$ to $0.04 \mathrm{~cm}^{-3}$ (Figure 2). We simulate one octant of the remnant, for the total time of 200 years, with resolution of 2048 $\times 512 \times 512$ grid cells, respectively per each of spherical coordinates $r, \theta$ and $\phi$ (Figure 3). Soon after the explosion, SNR dynamical evolution is characterized by increasing radius and decreasing shock velocity ${ }^{9}$. Observed shock velocity of $14000 \mathrm{~km} / \mathrm{s}$ and SNR radius of $2 \mathrm{pc}$ has to be reached in our simulations at the same time after the explosion, which we take as the SNR age. Hydrogen number density satisfying this requirement is $n_{\mathrm{H}}=0.02 \mathrm{~cm}^{-3}$ and corresponding SNR age is 115 years, for the epoch 2008 when observations, we used for comparison with models, are made. This implies an explosion date of about 1893 and current age of 123 years.

As a referent radio data for SNR G1.9+0.3 we use observations made by Green et al. (2008) at 1.43 and 4.86 $\mathrm{GHz}$ with the Very Large Array (VLA), which are respectively $0.935 \pm 0.047 \mathrm{Jy}$ and $0.437 \pm 0.022 \mathrm{Jy}$. Combining these integrated flux densities gives a steep radio emission spectral index, $\alpha$ (defined so that flux density scales with frequency as $\left.S_{v} \propto v^{-\alpha}\right)$ of $0.62 \pm 0.06$, using the

9 Note that shock velocity is not the fluid speed of any material present in our simulation box, but an interface between different conditions that is propagating through the fluid. 


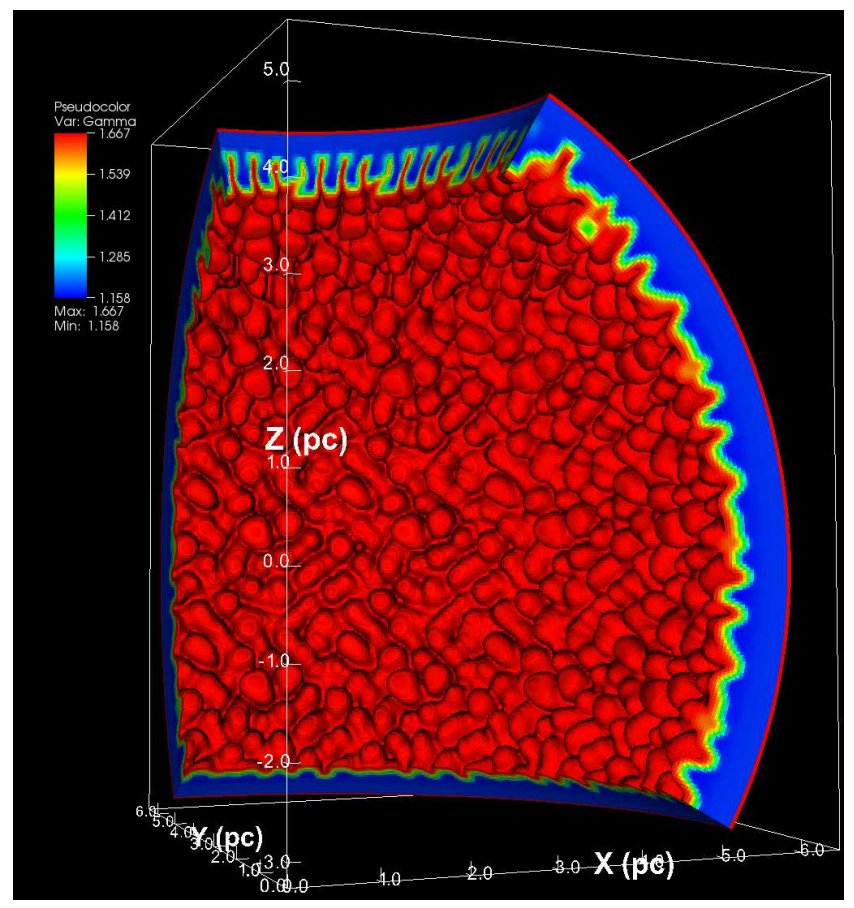

Figure 3. 3D rendering of the spatial distribution of plasma effective adiabatic index for a model accounting for the shock modification by accelerated CRs. Simulation describes the expansion of SNR through the interstellar medium. Credit by VisIt

assumed 5 per cent statistical uncertainties in the individual flux densities. In general, observations confirm that young SNRs have radio spectral indices steeper than the expected $\alpha=0.5$ (Urošević 2014), derived from TP DSA (Bell 1978a,b). Bell et al. (2011) showed that young SNRs with the quasi-perpendicular orientation of the magnetic field should have steeper spectral indices. For a detailed review on radio spectra of SNRs and some other possible explanations for steep spectra of young SNRs, see Urošević (2014) and references therein. Some properties of the time-dependent solutions (instead of quasi-stationary solutions used in our modeling) could also be responsible for deviation of the observed radio index from the classical value 0.5 in some young SNRs, as recently shown by Petruk \& Kopytko (2016).

We also poses a radio light curve for G1.9+0.3, observed with the Molonglo Observatory Synthesis Telescope (MOST), spanning $20 \mathrm{yr}$ from 1988 to 2007 at frequency of $843 \mathrm{MHz}$ (Murphy et al. 2008). Two most recent measurements (closest to the time when VLA observations have been made), $0.97 \pm 0.11$ Jy from epoch 2007.430 and 1.32 \pm 0.09 Jy from epoch 2007.463, will only be used for comparison with our best-fitting modeled spectra made using VLA measurements (Green et al. 2008), although showing evident inconsistency and large measurement errors. Change in radio flux should not be neglected for earlier MOST measurements, as they cover around one sixth of the estimated lifetime of the SNR.

De Horta et al. (2014) also obtained radio flux density measurements but significantly smaller $(\sim 50 \%)$ than VLA measurements. They attribute this large difference to missing short spacings and poorer $u v$ coverage of the ATCA images.

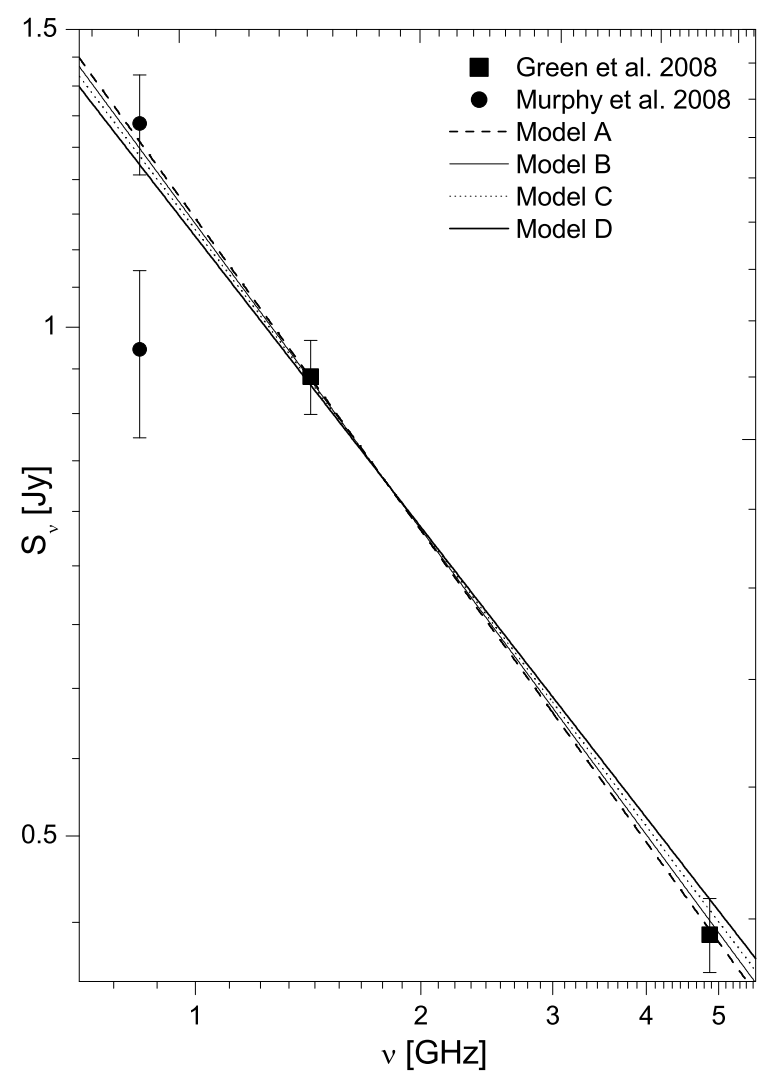

Figure 4. Measured radio fluxes at $843 \mathrm{MHz}$ (Murphy et al. 2008, MOST), $1425 \mathrm{MHz}$ and $4860 \mathrm{MHz}$ (Green et al. 2008, VLA); and four modelled radio spectra, corresponding to models from Table 1. We include only two MOST fluxes at $843 \mathrm{MHz}$ for comparison, observed in epochs 2007.46 and 2007.43, closest to the VLA observing epoch of 2008. Change in radio flux should not be neglected for earlier MOST measurements. Ambient magnetic field is set to value $B_{0}=5 \mu \mathrm{G}$. Observed and modeled radio fluxes corresponds to epoch 2008, when G1.9+0.3 was around 115 years old, as obtained from our simulations.

Our hydrodynamical approach does not selfconsistently deal with the magnetic turbulence and belonging higher order anisotropies which, according to Bell et al. (2011), steepen the spectral index at quasiperpendicular shocks. Due to this, we fit SNR G1.9+0.3 radio spectra simply by using described non-linear acceleration model of Blasi and assuming efficient acceleration (namely, acceleration efficiency up to $\eta \approx 10^{-3}$, to which corresponds $\xi$ between 3.3 and 3.45 in our simulations). Efficient acceleration is expected for such a young SNR and consistent with previous works (e.g., Ksenofontov et al. 2010). The electrons which mainly produce radiation by the synchrotron mechanism (in amplified magnetic field $\sim$ $100 \mu \mathrm{G}$ ) at frequencies $\sim 1 \mathrm{GHz}$ have energy $\sim 1 \mathrm{GeV}$ and momentum $\sim m_{p} c$. At energies around $1 \mathrm{GeV}$, our energy spectra $N(E) \propto E^{-\gamma}$ of accelerated particles at CR modified shocks become softer, with the effective power-law index $\gamma$ around 2.2, giving the required spectral steepening of synchrotron spectra.

From the fit of the synchrotron emission, we obtain value $2 \times 10^{-3}$ for electron-to-proton ratio $K_{\mathrm{ep}}$, which is slightly lower that the value observed in the local 
Table 1. Model parameters for simulated radio spectra. In all models ambient density was $0.02 \mathrm{~cm}^{-3}, \alpha$ represents radio emission spectral index defined in Section 3, $\eta$ stands for the fraction of particles entering acceleration (defined in Section 2.2), $S_{1.425}$ and $S_{4.860}$ are respectively modeled flux densities at 1430 and $4860 \mathrm{MHz}$ in Jy.

\begin{tabular}{ccccccccc}
\hline & $\xi$ & $\zeta$ & $\eta\left[\times 10^{-3}\right]$ & $\alpha$ & $S_{1.425}$ & $S_{4.860}$ & $R_{\text {tot }}$ & $R_{\text {sub }}$ \\
\hline Model A & 3.30 & 0.40 & 1.1 & 0.619 & 0.933 & 0.440 & 12.0 & 3.2 \\
Model B & 3.35 & 0.33 & 0.9 & 0.605 & 0.931 & 0.446 & 11.7 & 3.3 \\
Model C & 3.40 & 0.24 & 0.7 & 0.591 & 0.928 & 0.452 & 11.3 & 3.4 \\
Model D & 3.45 & 0.10 & 0.5 & 0.574 & 0.924 & 0.459 & 10.8 & 3.5 \\
\hline
\end{tabular}

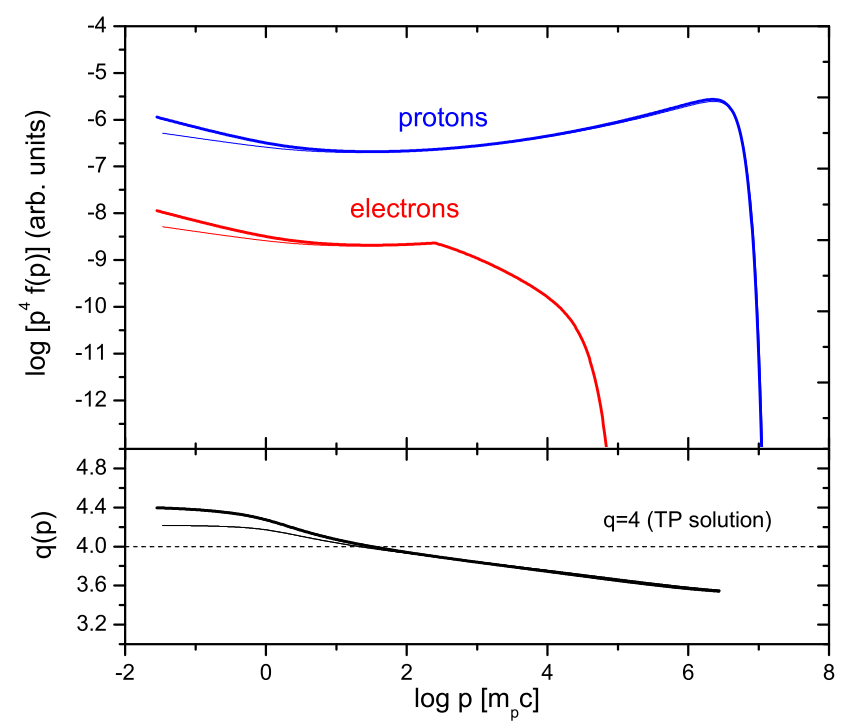

Figure 5. Cosmic ray electron and proton non-thermal part of the spectra (top panel) at the age of 115 years after explosion and proton spectra slope $q(p)=-\frac{d \ln f_{\mathrm{p}, 0}(p)}{d p}$ assuming particle distribution $\propto p^{-q}$ (bottom panel). Two representative models are shown, the most efficient NLDSA in Model A (thick solid line) and the least efficient NLDSA, among four models from Table 1, in Model $\mathrm{D}$ (thin solid line).

CR spectra. Our value is in good agreement with values derived by other authors (e.g., Ksenofontov et al. 2010; Morlino \& Caprioli 2012; Slane et al. 2014), although Yuan et al. (2012) proposed a model in which $K_{\mathrm{ep}}=10^{-2}$ is a universal value that can fit all Galactic SNRs. As the dynamical role of electrons is negligible in used model for NLDSA, this parameter acts as scaling factor for the total radio emission and should not have any qualitative effects on radio evolution.

Two free parameters are of the utmost importance for the total radio flux in our simulations: injection parameter $\xi$, determining a fraction of particles $\eta$ entering the acceleration process and therefore global efficiency of NLDSA and MFA; and Caprioli's parameter $\zeta$, controlling heating of the plasma by non-linear damping of Alfvén waves and therefore being able to reduce the shock modification to some extent.

As already said, efficient acceleration is necessary for the required spectral steepening of the radio spectra, but also $\xi$ values around 3.4 lead to very strong MFA and in turn can cause overestimate of SNR total radio flux. Therefore, some damping is likely and a search through parameter space leads to conclusion that, in our modeling, Caprioli's parameter $\zeta$ between 0.1 and 0.4 provide good fits. These values are also in agreement with Kang et al. (2013), who arbitrarily set $\zeta=0.5$ in their four heuristic models of MFA in the precursor and which is, according to them, a reasonable estimate. We choose three best-fitting models, for injection parameter $\xi$ values $3.30,3.35,3.40$ and 3.45 , respectively denoting them with Model A, B, C and D (Table 1 and Figure 4). We allow four different scenarios, although producing similar radio spectra for particular 2008. epoch, in order to allow possible differences in simulations of radio flux temporal evolution, being the main goal of our paper. Figure 4 indicate that MOST measurement $0.97 \pm$ 0.11 Jy should be taken with caution as probably being subject to significant measurement errors. In order to reproduce observed radio flux spectra from 2008, our simulations predict that amplified magnetic field in downstream was then around $B_{2}=280 \mu \mathrm{G}$. This value is in agreement with value $\approx 230 \mu \mathrm{G}$ inferred for G1.9+0.3 from equipartition calculations (Arbutina et al. 2012) and rapid variability in X-rays for notably older SNR RX J1713.72-3946, indicating amplification of the magnetic field by a factor of even more than 100 (Uchiyama et al. 2007). From the simulated 2500 years of evolution, the obtained amplified magnetic field in the downstream is $B_{2} \propto u_{0}^{0.76} \approx u_{0}^{3 / 4}$ (see Section 2.3 for discussion).

Figure 5 clearly shows that particle spectrum of accelerated particles changes from that predicted by standard linear DSA, which reads $\propto p^{-4}$ in momentum and corresponds to energy distribution $\propto E^{-2}$ in relativistic regime. Similarly to the cut-off for the electrons (Section 2.4), we parameterize the turnover of protons also by multiplying their distribution by an exponential factor, in a form suggested by Lee et al. (2012). For sufficiently large momenta, electron distribution function additionally deviate from the spectrum predicted by NLDSA, as a result of synchrotron losses.

As we mentioned before, a radio light curve for G1.9+0.3 based on 25 epochs of observation with the MOST is available (Murphy et al. 2008). These observations were taken with the same instrument, at constant frequency (843 $\mathrm{MHz})$ and comparable resolutions $(43 \times 91$ or $43 \times 95$ $\left.\operatorname{arcsec}^{2}\right)$. Therefore, we run our numerical simulations for different model parameters shown in Table 1 and synthesize total radio flux density at frequency $843 \mathrm{MHz}$ during the period from 1985 until 2010, in order to make comparison with observations (Figure 6). We obtained the average flux increase gradient during this period of around 0.0173 $\mathrm{Jy} \mathrm{yr}^{-1}\left(1.8\right.$ per cent $\left.\mathrm{yr}^{-1}\right)$, which is in a very good agreement with a least-squares fit of MOST observations which gives $0.015 \mathrm{Jy} \mathrm{yr}^{-1}\left(1.22 \pm_{0.16}^{0.24}\right.$ per cent $\left.\mathrm{yr}^{-1}\right)$ and also very close to the estimate of $\sim 2$ per cent $\mathrm{yr}^{-1}$ made by Green et al. 


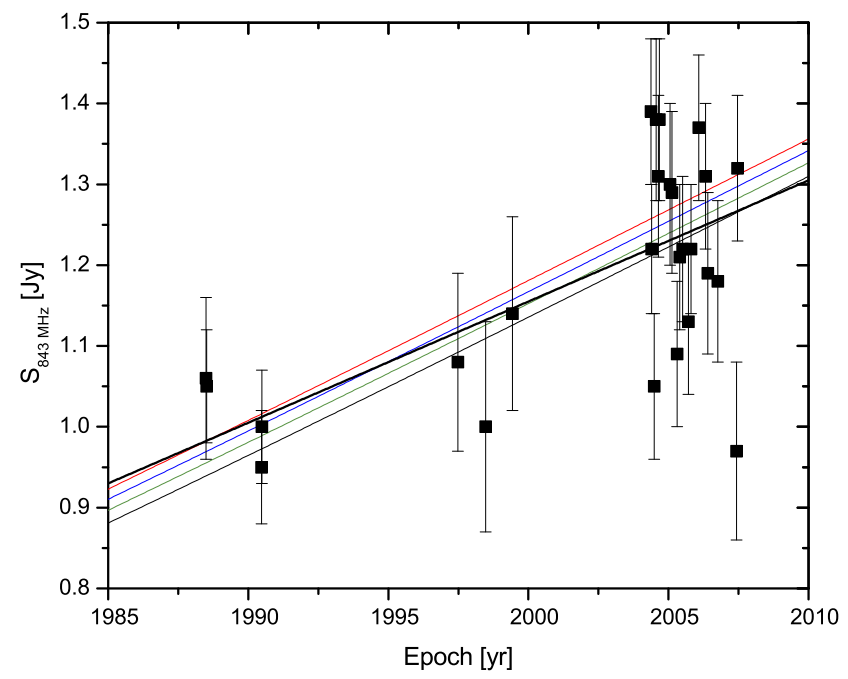

Figure 6. Observed and simulated evolution of integrated radio flux density at $843 \mathrm{MHz}$. Black squares with error bars represent radio light curve for G1.9+0.3 from 1988 to 2007, observed by MOST (Murphy et al. 2008). The black thick line shows a least-squares fit with gradient $0.015 \mathrm{Jy} \mathrm{yr}^{-1}$ and a flux density of 1.23 Jy on 2005 January 1, originally obtained by Murphy et al. (2008). Our simulations are shown with thin colour lines, namely Model A (red), Model B (blue), Model C (green) and Model C (black), showing similar average flux gradient $\approx 0.017 \mathrm{Jy} \mathrm{yr}^{-1}$

(2008), based on observations from a range of instruments, compiled from the literature. During the simulated part of the free expansion phase, derived dependence of radio flux density is $S_{V} \propto D^{2.675}$, corresponding to radio surface brightness dependence $\Sigma_{V} \propto D^{0.675}$, while Berezhko \& Völk (2004) derive steeper dependence $\Sigma_{v} \propto D$ in free expansion. Interestingly, X-ray flux brightening of G1.9+0.3, measured by Carlton et al. (2011), is also close to simulated and observed radio values, namely $1.7 \pm 1.0$ per cent $\mathrm{yr}^{-1}$.

We are also able to deduce forward shock expansion rate from our simulations, for the evolution period from 1985 until 2010, roughly covering available observations. Our value of 0.9 per cent $\mathrm{yr}^{-1}$ is in good agreement with expansion rate measurements $\approx 0.65$ per cent $\mathrm{yr}^{-1}$, from VLA observations (Green et al. 2008), and $0.642 \pm 0.049$ per cent $\mathrm{yr}^{-1}$ (Carlton et al. 2011), obtained by comparing Chandra X-ray images.

In Figure 7, we plot time evolution of different characteristics of SNR for period of 1000 years and compare the evolution corresponding to efficient DSA, including MFA, with a TP case. Red line represents DSA with pronounced non-linear effects and strongly modified shock (parameters from Model A), while blue line represents SNR evolution with injection parameter $\xi=4.8$ leading to almost negligible amount of accelerated CRs. Total compression in TP case reduces to 4 and magnetic field downstream is amplified only due to gas compression, which is far bellow the value required for the observed radio emission.

Being the main purpose of this paper, we simulate the time dependence of flux densities $S_{v}$ of the radio synchrotron emission at different frequencies with its corresponding rate of change $S_{V}$ in Jy per year and frequency independent fractional change $\frac{S_{v}}{S_{v}}$ in percentage per year (Figure 8). Our

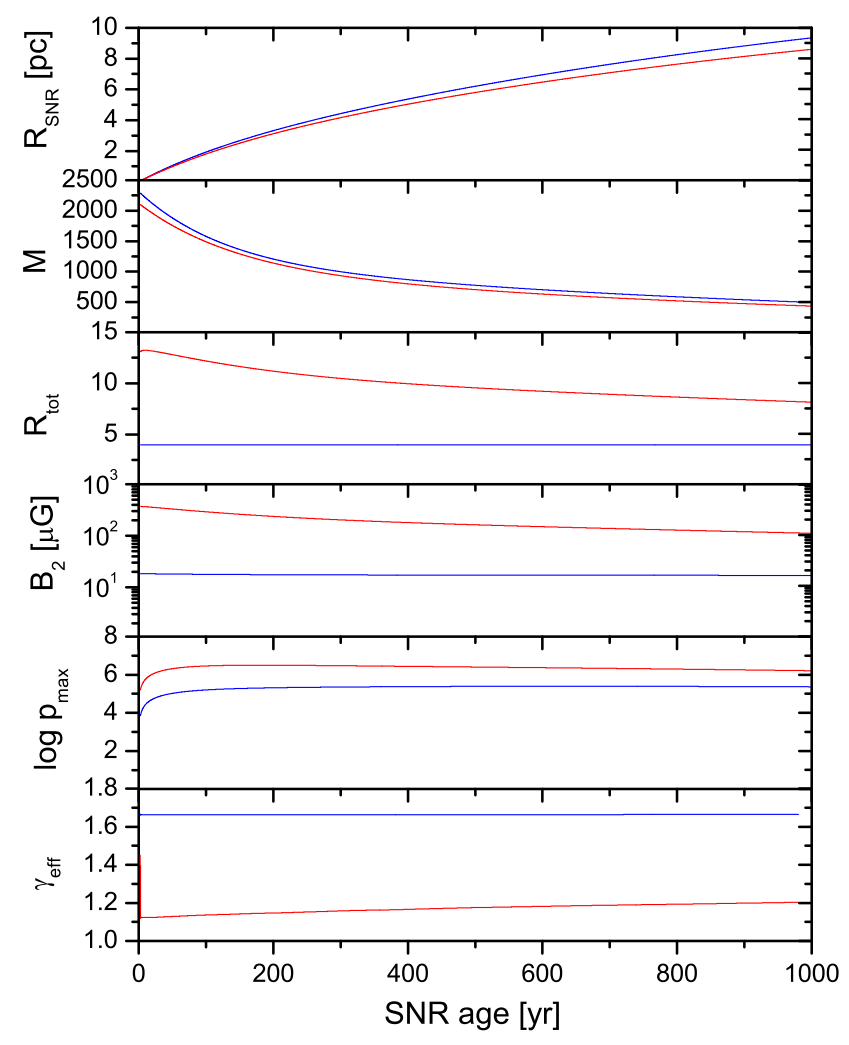

Figure 7. Evolution parameters of G1.9+0.3 for period of 1000 yr. Blue line represents TP DSA, namely for $\xi=4.8$ and red line represents NLDSA with Model A parameters (the most efficient acceleration among models listed in Table 1).

model predicts increasing radio emission from G1.9+0.3 during the part of free expansion phase, reaching its maximum value around the age of 600 years and then decreasing during later free expansion and Sedov phase. For determined ambient density of $0.02 \mathrm{~cm}^{-3}$, radius around $11.3 \mathrm{pc}$ marks the end of free expansion (ejecta dominated) phase, which we assume to be when the swept-up mass is $M_{\mathrm{sw}}=3 M_{\mathrm{ej}}$. In our simulations, this happens $\approx 1700$ years after the SN explosion. Model also predicts maximum radio flux densities $\sim 4.3,3.9$ and $3.1 \mathrm{Jy}$, respectively for frequencies 843, 1000 and $1425 \mathrm{MHz}$, being around 3 times higher than present values. It can be inferred from Figure 8 that models with higher injection parameter $\xi$ (less efficient acceleration) give slightly higher flux densities close to maximum, for a chosen frequency. This is mainly due to the increasing efficiency of MFA in Models from A to D (see Table 1), linked with the parameter $\zeta$. As for the rate of flux density change during time (in Jy per year), our model suggests a maximum at around $t_{\mathrm{SNR}} \sim 100 \mathrm{yr}$ followed by gradual slowing down of flux increase, until it starts to decline. Interestingly, available measurements of radio light curve for G1.9+0.3 roughly coincide with this maximum (Green et al. 2008; Murphy et al. 2008; Carlton et al. 2011), meaning they probably contain the fastest ever flux change for this SNR.

Simulations also give insight into the radio spectral index $\alpha$ evolution (Figure 9), reflecting the evolution of the spectrum of accelerated electrons with energies around $\sim$ $\mathrm{GeV}$. Evolution starts from the values close to the $\alpha=0.5$, corresponding to TP DSA solution, reaches maximum value 


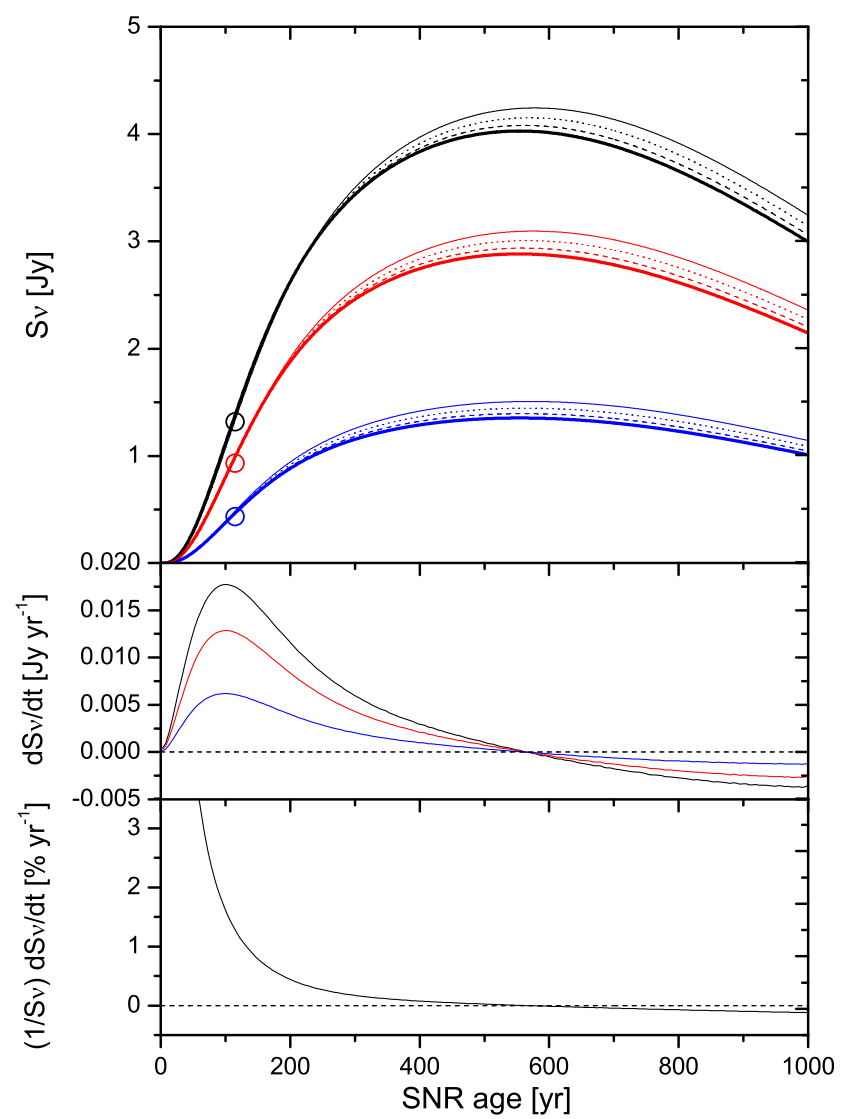

Figure 8. Simulated radio evolution of G1.9+0.3 during 1000 years after SN explosion. Upper panel shows radio flux density evolution at three frequencies: $843 \mathrm{MHz}$ (black lines), $1000 \mathrm{MHz}$ (red) and $1425 \mathrm{MHz}$ (blue). Four models are shown for each frequency: Model A (thick solid line), Model B (dashed), Model C (dotted) and Model D (thin solid). Open circles denote radio flux densities observed in 2008. Middle panel shows corresponding rate of change of flux density $\frac{d S_{v}}{d t}$ in Jy per year, averaged for each frequency and line colours having the same meaning as in the upper panel. Lower panel shows annual flux density increase, $\frac{1}{S_{v}} \frac{d S_{V}}{d t}$, in \% per year, which is independent of frequency. Maximum flux density is predicted around $600 \mathrm{yr}$, which is before the shock sweeps ISM mass equal to $3 M_{\odot}$, roughly marking the end of free expansion phase in our simulation $(\approx 1700 \mathrm{yr})$.

(the steepest radio spectra) and then slowly decreases, making spectra shallower. Evolutionary tracks for radio spectral index were obtained by implementing models from Table 1 and they seem strongly model dependent. Higher injection efficiency (lower $\xi$ parameter) naturally leads to higher value for $\alpha$ in maximum but also this maximum is reached earlier in the SNR lifetime. This is in good agreement with a considerable amount of observational evidence for steep spectral indices of SNRs being $\sim 1000$ years old or even few times older, as radio spectral index slowly decrease after reaching maximum steepness. The greatest number of evolved SNRs have spectral indices in the interval $0.5 \leq \alpha \leq 0.6$, as the DSA predicts (Urošević 2014).

Synchrotron emission spans from the radio to the X-ray band. Reynolds et al. (2008) concluded that X-ray emission from G1.9+0.3 appears to be purely synchrotron radiation. We neglect X-ray emission due to thermal bremsstrahlung

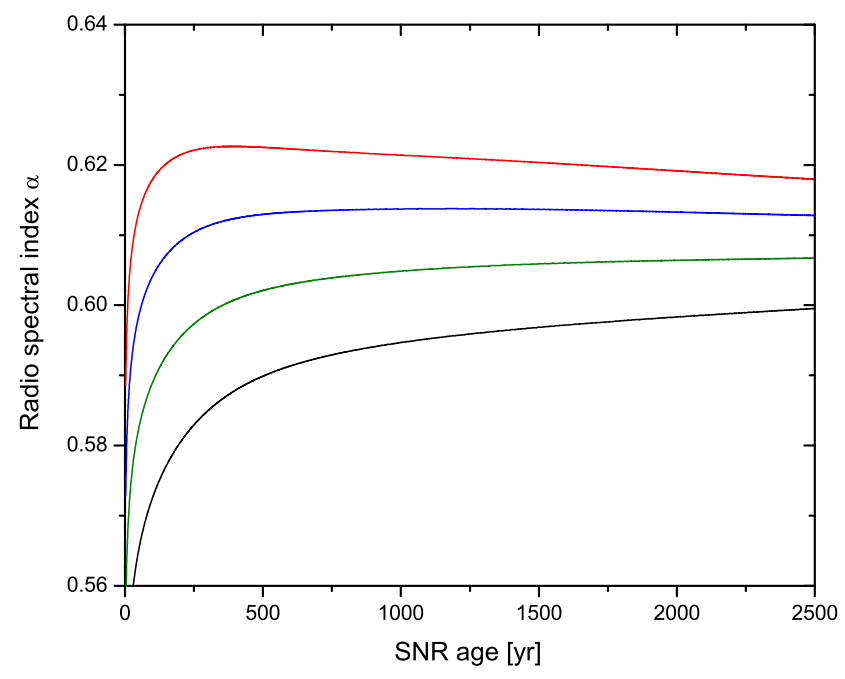

Figure 9. Simulated evolution of the radio spectral index $\alpha$, defined so that flux density depends on frequency as $S_{v} \propto v^{-\alpha}$. Spectral index evolution is model dependent and due to this we denote different scenarios with colours, namely Model A (red line), Model B (blue), Model C (green) and Model D (black).

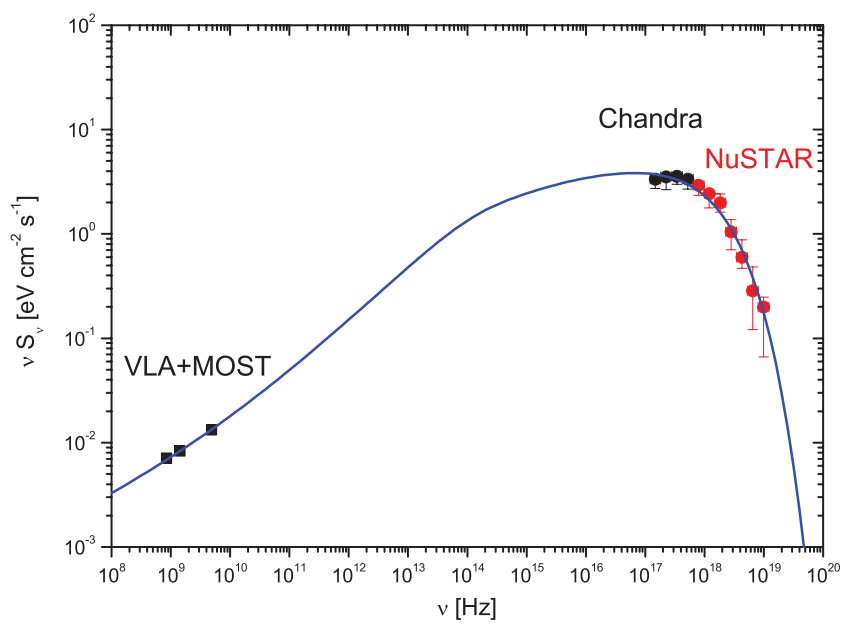

Figure 10. Spatially integrated synchrotron flux density as a function of frequency from radio to X-ray domain. The observational data are, respectively: radio from VLA (Green et al. 2008) and MOST (Murphy et al. 2008); X-ray fluxes are taken from Yang et al. (2016), originally observed with Chandra (Reynolds et al. 2008) and NuSTAR (Zoglauer et al. 2015) telescopes.

and synthesize synchrotron spectrum up to the highest energies by using electron spectrum obtained in our simulations (Figure 10) and adopted to fit observations, as described in Section 2.4. Magnetic field $B_{2}=280 \mu \mathrm{G}$ inferred from our simulations for SNR age of $t_{0}=115 \mathrm{yr}$ in 2008, gives the position of the break in the electron spectrum around $p_{b} \approx 10^{3} m_{p} c$ (Equation 16). The best-fitting maximum value for the steepening $\delta$ is 0.5 , implemented through our "toy" model (Section 2.4) as uniformly growing function starting from momenta slightly before $p_{b}$, to assure physically more consistent and smoother transition from uncooled to cooled regime instead of a sharp break in the electron spec- 


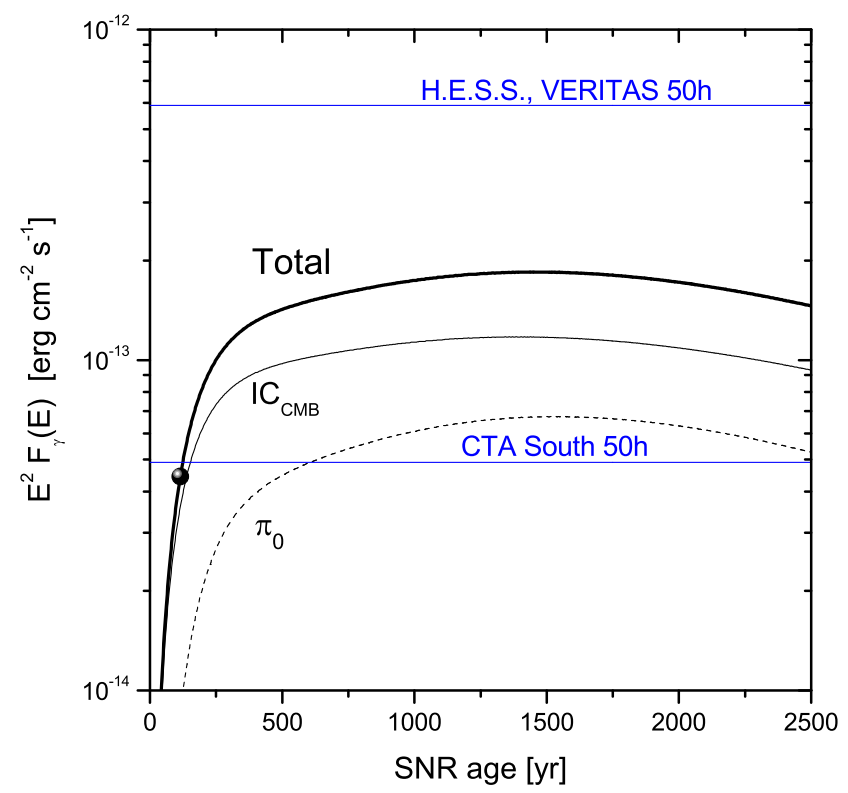

Figure 11. Evolution of spatially integrated $4 \mathrm{TeV}$ gamma-ray emission produced by pion decay (dashed line) and IC computed for the CMB photon field (thin solid line). The thick solid line is the sum of these two contributions. Blue lines represents corresponding flux sensitivities of H.E.S.S., VERITAS and CTA gamma telescopes. Black ball represents the current evolutionary status.

trum $^{10}$. The synchrotron spectrum in Figure 10 is obtained only for Model A and it is obtained for maximum electron cut-off momentum $p_{\mathrm{e}, \max }=10^{4.5} m_{\mathrm{p}} c$ and corresponding energy $E_{\mathrm{e}, \max }=27 \mathrm{TeV}$, obtained by assuming Bohm diffusion. Spectra for the remaining three models (Table 1) were omitted because they are very similar. Also, modeled spectra of G1.9+0.3 reveals concave-up curvature at millimeter and sub-millimeter wavelengths. This is expected and indeed observed by Planck ${ }^{11}$ telescope in the radio continuum of another young SNR Cas A (Onić \& Urošević 2015). After investigating alternative explanations of the observed curvature, Onić \& Urošević (2015) agree that non-linear effects of particle acceleration are mainly responsible for highfrequency curvature in radio spectrum.

Following the approach described in Section 2.5, we evolve the SNR during the 2500 years and calculate its 4 $\mathrm{TeV}$ gamma ray emission produced by pion decay and IC computed for the CMB photon field (Figure 11). We chose to model $4 \mathrm{TeV}$ emission intentionally, as the highest CTA sensitivity is expected around this energy ${ }^{12}$. For the present SNR age, the expected total $\mathrm{TeV}$ gamma-ray emission is $4.4 \times 10^{-14} \mathrm{erg} \mathrm{cm}^{-2} \mathrm{~s}^{-1}$. Such a flux is too low for possi-

10 Tanaka et al. (2008) mention possible detection of this synchrotron break in optical/infrared wavelengths as additional argument in favor of strong MFA.

11 A project of the European Space Agency (ESA). It observes the sky in nine frequency bands covering 30-857 GHz with high sensitivity and angular resolution.

12 Reference: CTA energy flux sensitivity, www.cta-observatory.org ble H.E.S.S. ${ }^{13}$ or VERITAS ${ }^{14}$ detection in $\sim 50 \mathrm{hr}$, more than one order of magnitude bellow their sensitivities. From the other side, present value from our model is slightly bellow the predicted CTA (Southern Site) sensitivity limit of $4.9 \times 10^{-14} \mathrm{erg} \mathrm{cm}^{-2} \mathrm{~s}^{-1}$ around $\mathrm{TeV}$ energies, but expected to reach this limit within a decade or so. The pion decay flux is only about $1 / 4$ of the IC gamma-ray flux, probably as a result of the low ambient gas density. Maximum $\mathrm{TeV}$ gamma-ray flux is predicted to occur around the end of free expansion phase, at the age of $1500 \mathrm{yr}$, and it reaches $1.8 \times 10^{-13} \mathrm{erg} \mathrm{cm}^{-2} \mathrm{~s}^{-1}$. This value is still bellow the sensitivity limit of H.E.S.S., but probably visible in $\mathrm{TeV}$ gammarays by future instruments, including the CTA.

However, more advanced broadband modeling of G1.9+0.3 is out of a scope of this paper. X-ray part of the spectrum and gamma-ray emission evolution are given above only in an illustrative way, to check how our model fits with observations in domains other than radio. More rigorous numerical treatment of synchrotron loses will be necessary in order to obtain evolution of the emission at energies higher than radio. We reserve a detailed modeling of SNR evolutionary tracks at different wavelengths for future work.

\section{CONCLUSION}

The peculiar nature of radio evolution of the youngest known Galactic SNR G1.9+0.3 is modeled by using Blasi non-linear kinetic theory of CR acceleration in SNRs coupled with 3D hydrodynamics, simultaneously solved with PLUTO code. We assume this SNR originated from a type Ia supernova explosion located near the Galactic centre, with explosion energy $10^{51} \mathrm{erg}$ and ejecta mass $1.4 \mathrm{M}_{\odot}$. Hydrodynamic equations in PLUTO code were adopted to use the space and time-dependent adiabatic index in order to account for the presence of energetic particles, making the fluid more compressible. Our modeling and analysis leads to the following essential results:

(i) From our 3D hydrodynamic simulations of SNR evolution, including a deceleration of forward shock by the ambient medium and due to back reaction of CRs, we estimate the current age of G1.9+0.3 SNR to be slightly over 120 years, expanding in ambient density of $0.02 \mathrm{~cm}^{3}$.

(ii) Efficient acceleration is necessary in order to explain observed spectral steepening of the radio spectra. Namely, observations are well fitted for injection parameter $\xi$ between 3.45 and 3.30, corresponding to an acceleration efficiency $\eta=(0.5-1.1) \times 10^{-3}$ and magnetic field amplified more than 50 times from the assumed ambient value.

(iii) Following our models, it can be concluded that radio emission increasing brightness is a common property of young SNRs. Our model gives the average $843 \mathrm{MHz}$ flux increase gradient during 20-year period of around 0.0173 $\mathrm{Jyyr}^{-1}\left(1.8\right.$ per cent $\left.\mathrm{yr}^{-1}\right)$, which is in a very good agreement with MOST observations and also with other available

13 H.E.S.S.: Preliminary sensitivity curves for H.E.S.S.-I (stereo reconstruction), adapted from Holler et al. (2015)

14 Very Energetic Radiation Imaging Telescope Array System (VERITAS): public specifications webpage veritas.sao.arizona.edu/about-veritas-mainmenu-81/veritas-specificat 
observations from a range of instruments, compiled from the literature.

(iv) Numerical model predicts increasing radio emission from G1.9+0.3 during the free expansion phase, reaching its maximum value around the age of 600 years and then decreasing during late free expansion and beginning of Sedov phase around 1700 years after the SN explosion. Interestingly, it seems that we are currently witnessing approximately the fastest radio emission increasing than it will ever be.

(v) The radio brightness will grow according to prediction given in this paper, until its maximum flux densities of $\sim 4.3,3.9$ and $3.1 \mathrm{Jy}$, respectively for frequencies 843, 1000 and $1425 \mathrm{MHz}$, being around 3 times higher than the present day values.

(vi) The steep radio spectral index (steeper than linear DSA prediction of $\alpha=0.5$ ) for young SNRs is explained only by means of efficient NLDSA and accompanying strong MFA. Radio spectral index also shows qualitatively similar evolution as the radio flux, it reaches the steepest value $\alpha_{\max }$ and then becomes shallower (trending towards the value of $0.5)$. Higher injection efficiency $\eta$ leads to higher $\alpha_{\max }$ but also causes this value to be reached earlier in the SNR history. However, temporal evolution of radio spectral index turns out to be very sensitive to model parameters $\xi$ and $\zeta$.

(vii) We implement a simple "toy" model for synthesis of broader synchrotron spectrum from radio to X-ray, by using electron spectrum obtained in our simulations. This spectrum is modified in post processing by introducing a break in the electron spectrum, to account for synchrotron losses and modeled X-ray emission fits well the Chandra and NuSTAR measurements. It agrees well with models of spectra containing more consistent, numerical calculation of synchrotron losses.

(viii) We also implement approximative model of gamma-ray emission coming from the SNR. We inspect time evolution of the total gamma ray flux and conclude it may be visible in $\mathrm{TeV}$ gamma-rays by future instruments, including the CTA. Model predicts increasing TeV gamma-ray emission during entire free expansion phase, reaching the maximum value of $1.8 \times 10^{-13} \mathrm{erg} \mathrm{cm}^{-2} \mathrm{~s}^{-1}$ at the age of around $1500 \mathrm{yr}$.

Our model enabled us to make important conclusions about present and predictions about future properties of radio emission from the youngest known Galactic SNR. We want to emphasize that, although presented model contain robust implementation, all provided quantitative estimates should be taken with caution. Besides our limited knowledge in physical descriptions of particle acceleration and SNR evolution, a significant number of model parameters still remain weakly constrained.

Models of radio evolutionary tracks can be of the utmost importance for the future observers working on powerful radio telescopes like ALMA ${ }^{15}$ and $\mathrm{SKA}^{16}$. These type of modeling can provide important information about the evolutionary stage of SNRs, as well as to characterize the physical conditions in the shocks where the relativistic particles are accelerated.

15 The Atacama Large Millimeter/submillimeter Array

16 The Square Kilometre Array

\section{ACKNOWLEDGEMENTS}

This work is part of Project No. 176005 "Emission nebulae: structure and evolution" supported by the Ministry of Education, Science, and Technological Development of the Republic of Serbia. Numerical simulations were run on the PARADOX-IV supercomputing facility at the Scientific Computing Laboratory of the Institute of Physics Belgrade, supported in part by the Ministry of Education, Science and Technological Development of the Republic of Serbia under project No. ON171017. Simulations were also run on cluster Jason, belonging to Automated Reasoning Group (ARGO) based at the Department of Computer Science, Faculty of Mathematics, University of Belgrade. I thank the anonymous referee for the very constructive suggestions on this manuscript. I would like to thank D. Urošević and B. Arbutina for introducing me into this exciting field, continually supporting and encouraging me, but also for careful reviewing and editing of typescript. I acknowledge the hospitality of the Osservatorio Astronomico di Palermo where part of this work was carried out, special thanks to Salvatore Orlando and Marco Micelli for their illuminating contributions to this project and also for reading the manuscript. I'm grateful to Gilles Ferrand for extremely helpful discussions, advices and help during this work and coding. Brian Reville provided valuable discussion on different approaches in SNR modeling. I'm indebted to Tara Murphy for kindly providing of currently available MOST radio flux densities and to RuiZhi Yang and Leonid Ksenofontov for sharing the X-ray data for G1.9+0.3 and useful explanations. PLUTO is developed at the Turin Astronomical Observatory in collaboration with the Department of Physics of the Turin University.

\section{REFERENCES}

Abramowitz, M., \& Stegun, I. A. 1972, Handbook of Mathematical Functions, New York: Dover Publications

Amato, E., \& Blasi, P. 2005, MNRAS, 364, L76

Amato, E., \& Blasi, P. 2009, MNRAS, 392, 1591

Amato, E. 2011, Mem. Soc. Astron. Italiana, 82, 806

Arbutina, B., \& Urošević, D. 2005, MNRAS, 360, 76

Arbutina, B., Urošević, D., Andjelić, M. M., Pavlović, M. Z., \& Vukotić, B. 2012, ApJ, 746, 79

Beck, R., \& Krause, M. 2005, Astronomische Nachrichten, 326, 414

Bell, A. R. 1978a, MNRAS, 182, 147

Bell, A. R. 1978b, MNRAS, 182, 443

Bell, A. R. 2004, MNRAS, 353, 550

Bell, A. R. 2005, MNRAS, 358, 181

Bell, A. R., Schure, K. M., \& Reville, B. 2011, MNRAS, 418, 1208

Bell, A. R., Schure, K. M., Reville, B., \& Giacinti, G. 2013, MNRAS, 431, 415

Berezhko, E. G., \& Ellison, D. C. 1999, ApJ, 526, 385

Berezhko, E. G., Ksenofontov, L. T., \& Völk, H. J. 2002, A\&A, 395,943

Berezhko, E. G., Völk, H. J. 2004, A\&A, 427, 525

Blandford, R. D., \& Ostriker, J. P. 1978, ApJ, 221, L29

Blasi, P. 2002, Astroparticle Physics, 16, 429

Blasi, P. 2004, Astroparticle Physics, 21, 45

Blasi, P., Gabici, S., \& Vannoni, G. 2005, MNRAS, 361, 907

Blasi, P., Amato, E., \& Caprioli, D. 2007, MNRAS, 375, 1471

Blasi, P. 2010, MNRAS, 402, 2807

Blondin, J. M., Wright, E. B., Borkowski, K. J., \& Reynolds, S. P. 1998, ApJ, 500, 342 
Borkowski, K. J., Reynolds, S. P., Green, D. A., et al. 2010, ApJ, 724, L161

Borkowski, K. J., Reynolds, S. P., Hwang, U., et al. 2013, ApJ, 771, L9

Brose, R., Telezhinsky, I., \& Pohl, M. 2016, A\&A, 593, A20

Caprioli, D., Blasi, P., Amato, E., \& Vietri, M. 2009, MNRAS, 395,895

Caprioli, D., Amato, E., \& Blasi, P. 2010, Astroparticle Physics, 33,307

Caprioli, D. 2012, J. Cosmology Astropart. Phys., 7, 038

Caprioli, D., \& Spitkovsky, A. 2014a, ApJ, 783, 91

Caprioli, D., \& Spitkovsky, A. 2014b, ApJ, 794, 46

Caprioli, D., Pop, A.-R., \& Spitkovsky, A. 2015, ApJ, 798, L28

Carlton, A. K., Borkowski, K. J., Reynolds, S. P., et al. 2011, ApJ, 737, L22

Chakraborti, S., Childs, F., \& Soderberg, A. 2016, ApJ, 819, 37

De Horta, A. Y., Filipovic, M. D., Crawford, E. J., et al. 2014, Serbian Astronomical Journal, 189, 41

Dwarkadas, V. V., \& Chevalier, R. A. 1998, ApJ, 497, 807

Ellison, D. C., Decourchelle, A., \& Ballet, J. 2004, A\&A, 413, 189

Ellison, D. C., Decourchelle, A., \& Ballet, J. 2005, A\&A, 429, 569

Fermi, E. 1949, Phys. Rev., 75, 1169

Ferrand, G., Decourchelle, A., Ballet, J., Teyssier, R., \& Fraschetti, F. 2010, A\&A, 509, L10

Ferrand, G., Decourchelle, A., \& Safi-Harb, S. 2012, ApJ, 760, 34

Ferrand, G., Decourchelle, A., \& Safi-Harb, S. 2014, ApJ, 789, 49

Fouka, M., \& Ouichaoui, S. 2013, Research in Astronomy and Astrophysics, 13, 680-686

Gotthelf, E. V., Koralesky, B., Rudnick, L., et al. 2001, ApJ, 552, L39

Green, D. A., \& Gull, S. F. 1984, Nature, 312, 527

Green, D. A. 2004, Bulletin of the Astronomical Society of India, 32,335

Green, D. A., Reynolds, S. P., Borkowski, K. J., et al. 2008, MNRAS, 387, L54

Helder, E. A., Vink, J., Bykov, A. M., et al. 2012, Space Sci. Rev., 173,369

Holler, M., Berge, D., van Eldik, C., et al. 2015, arXiv:1509.02902

H.E.S.S. Collaboration, Abramowski, A., Aharonian, F., et al. 2014, MNRAS, 441, 790

Kang, H., Jones, T. W., \& Edmon, P. P. 2013, ApJ, 777, 25

Kelner, S. R., Aharonian, F. A., \& Bugayov, V. V. 2006, Phys. Rev. D, 74, 034018

Ksenofontov, L. T., Völk, H. J., \& Berezhko, E. G. 2010, ApJ, 714,1187

Lee, S.-H., Ellison, D. C., \& Nagataki, S. 2012, ApJ, 750, 156

Longair, M. S. 2011, High Energy Astrophysics, Cambridge, UK: Cambridge University Press, 2011

Mignone, A., Bodo, G., Massaglia, S., et al. 2007, ApJS, 170, 228

Mignone, A., Zanni, C., Tzeferacos, P., et al. 2012, ApJS, 198, 7

Morlino, G., Amato, E., \& Blasi, P. 2009, MNRAS, 392, 240

Morlino, G., \& Caprioli, D. 2012, A\&A, 538, A81

Murphy, T., Gaensler, B. M., \& Chatterjee, S. 2008, MNRAS, 389, L23

Onić, D., \& Urošević, D. 2015, ApJ, 805, 119

Orlando, S., Bocchino, F., Reale, F., Peres, G., \& Petruk, O. 2007, A\&A, 470, 927

Orlando, S., Petruk, O., Bocchino, F., \& Miceli, M. 2011, A\&A, 526, A129

Orlando, S., Bocchino, F., Miceli, M., Petruk, O., \& Pumo, M. L. 2012, ApJ, 749, 156

Pavlovic, M. Z., Dobardzic, A., Vukotic, B., \& Urosevic, D. 2014, Serbian Astronomical Journal, 189, 25

Park, J., Caprioli, D., \& Spitkovsky, A. 2015, Physical Review Letters, 114, 085003

Petruk, O. 2005, Journal of Physical Studies, 9, 364

Petruk, O., Kopytko, B., 2016 (arXiv:1607.07206)
Reynolds, S. P., Borkowski, K. J., Green, D. A., et al. 2008, ApJ, 680, L41

Reynolds, S. P. 2008, ARA\&A, 46, 89

Reynolds, S. P. 2011, Ap\&SS, 336, 257

Reynolds, S. P., Gaensler, B. M., \& Bocchino, F. 2012, Space Sci. Rev., 166, 231

Roy, S., \& Pal, S. 2014, Supernova Environmental Impacts, 296, 197

Sarbadhicary, S. K., Badenes, C., Chomiuk, L., Caprioli, D., \& Huizenga, D. 2017, MNRAS, 464, 2326

Slane, P., Lee, S.-H., Ellison, D. C., et al. 2014, ApJ, 783, 33

Tanaka, T., Uchiyama, Y., Aharonian, F. A., et al. 2008, ApJ, 685, 988-1004

Uchiyama, Y., Aharonian, F. A., Tanaka, T., Takahashi, T., \& Maeda, Y. 2007, Nature, 449, 576

Urošević, D., Vukotić, B., Arbutina, B., \& Sarevska, M. 2010, ApJ, 719, 950

Urošević, D. 2014, Ap\&SS, 354, 541

Vink, J. 2012, A\&ARv, 20, 49

Völk, H. J., Berezhko, E. G., \& Ksenofontov, L. T. 2005, A\&A, 433, 229

Wilson, T. L., Rohlfs, K. \& Huettemeister, S., 2013, Tools of Radio Astronomy, Springer, Berlin

Yang, R.-z., Sun, X.-n., Aharonian, F., 2016 (arXiv:1612.02262)

Yuan, Q., Liu, S., \& Bi, X. 2012, ApJ, 761, 133

Zirakashvili, V. N., \& Aharonian, F. 2007, A\&A, 465, 695

Zirakashvili, V. N. 2008, American Institute of Physics Conference Series, 1085, 129

Zirakashvili, V. N., \& Aharonian, F. A. 2010, ApJ, 708, 965

Zirakashvili, V. N., \& Ptuskin, V. S. 2012, Astroparticle Physics, 39,12

Zoglauer, A., Reynolds, S. P., An, H., et al. 2015, ApJ, 798, 98 